\title{
Partial flag varieties and preprojective algebras
}

\author{
C. Geiß, B. Leclerc and J. Schröer \\ Dedicated to Toshiaki Shoji on the occasion of his sixtieth birthday.
}

\begin{abstract}
Let $\Lambda$ be a preprojective algebra of type $A, D, E$, and let $G$ be the corresponding semisimple simply connected complex algebraic group. We study rigid modules in subcategories $\operatorname{Sub} Q$ for $Q$ an injective $\Lambda$-module, and we introduce a mutation operation between complete rigid modules in $\operatorname{Sub} Q$. This yields cluster algebra structures on the coordinate rings of the partial flag varieties attached to $G$.
\end{abstract}

\section{Résumé}

Soit $\Lambda$ une algèbre préprojective de type $A, D, E$, et soit $G$ le groupe algébrique complexe semi-simple et simplement connexe correspondant. Nous étudions les modules rigides des sous-catégories $\operatorname{Sub} Q$ où $Q$ désigne un $\Lambda$-module injectif, et nous introduisons une opération de mutation sur les modules rigides complets de $\operatorname{Sub} Q$. Ceci conduit à des structures d'algèbre amassée sur les anneaux de coordonnées des variétés de drapeaux partiels associées à $G$.

\section{Contents}

1 Introduction 2

2 Coordinate algebras associated to parabolic subgroups 3

3 The category $\operatorname{Sub} Q_{J}$

4 The category $\left(\operatorname{Sub} Q_{J}\right)^{\perp}$

5 The functors $\mathscr{E}_{i}$ and $\mathscr{E}_{i}^{\dagger}$

6 Generalized minors and maximal rigid modules 13

7 Complete rigid modules in $\operatorname{Sub} Q_{J}$

8 Mutations in $\operatorname{Sub} Q_{J}$

9 Cluster algebra structure on $\mathbb{C}\left[N_{K}\right]$

10 Cluster algebra structure on $\mathbb{C}\left[B_{K}^{-} \backslash G\right]$

11 Finite type classification 29

12 The coordinate ring of a smooth quadric in $\mathbb{P}^{2 n-1}(\mathbb{C})$

13 Isotropic Grassmannians 36

14 Remarks on the non simply-laced case 


\section{Introduction}

Let $G$ be a simple simply connected complex algebraic group of simply-laced type $A, D, E$. Let $P$ be a parabolic subgroup of $G$. The projective variety $P \backslash G$ is called a partial flag variety. Let $\mathbb{C}[P \backslash G]$ be the multi-homogeneous coordinate ring of $P \backslash G$ for its Plücker embedding into a product of projective spaces.

When $P=B$ is a Borel subgroup with unipotent radical $N, \mathbb{C}[B \backslash G]$ is equal to the coordinate ring of the base affine space $\mathbb{C}[N \backslash G]$. Let $w_{0}$ be the longest element of the Weyl group $W$ of $G$. The double Bruhat cell $G^{e, w_{0}}$ [9] can be naturally identified to an open subset of $N \backslash G$. Berenstein, Fomin and Zelevinsky [4] have shown that $\mathbb{C}\left[G^{e, w_{0}}\right]$ has a nice cluster algebra structure, with initial seeds described explicitly in terms of the reduced expressions of $w_{0}$.

When $G=S L_{n}$ and $P$ is a maximal parabolic subgroup, $\mathrm{Gr}=P \backslash G$ is a Grassmann variety and Scott [32] has studied in detail a cluster algebra structure on $\mathbb{C}[\mathrm{Gr}]$ (see also [20], where a cluster algebra on an open Bruhat cell of $\mathrm{Gr}$ was previously introduced).

In [15], we considered a cluster algebra structure on $\mathbb{C}[N]$ coming from the one on $\mathbb{C}\left[G^{e, w_{0}}\right]$, and we showed that it is deeply connected to the representation theory of the preprojective algebra $\Lambda$ attached to the Dynkin diagram of $G$. We proved that every cluster of $\mathbb{C}[N]$ comes in a natural way from a complete rigid $\Lambda$-module and that the cluster mutation lifts to a new operation on complete rigid modules that we also call mutation. This allowed us to prove that every cluster monomial is an element of the dual semicanonical basis, and in particular that cluster monomials are linearly independent.

In this paper we consider an arbitrary partial flag variety $P \backslash G$. Let $N_{P}$ denote the unipotent radical of $P$. We introduce a cluster algebra $\mathscr{A} \subseteq \mathbb{C}\left[N_{P}\right]$ whose initial seed is described in terms of certain reduced expressions of $w_{0}$. We then lift $\mathscr{A}$ to a cluster algebra $\widetilde{\mathscr{A}} \subseteq \mathbb{C}[P \backslash G]$. We conjecture that in fact $\mathscr{A}=\mathbb{C}\left[N_{P}\right]$, and that $\widetilde{\mathscr{A}}$ coincides with $\mathbb{C}[P \backslash G]$ up to localization with respect to certain generalized minors (see 10.4 for a precise formulation). We give a proof of the conjectures in type $A$ and in type $D_{4}$. We also give a complete classification of the algebras $\mathscr{A}$ (or equivalently $\mathscr{A}$ ) which are of finite type as cluster algebras.

We arrived at the definition of $\mathscr{A}$ by studying certain subcategories of $\bmod \Lambda$. Let $Q$ be an injective $\Lambda$-module, and denote by $\operatorname{Sub} Q$ the full subcategory of $\bmod \Lambda$ whose objects are the submodules of a finite direct sum of copies of $Q$. This is a classical example of a homologically finite subcategory (see [1, 2]). It inherits from $\bmod \Lambda$ the structure of a Frobenius category. We show that the relative syzygy functor of $\operatorname{Sub} Q$ coincides with the inverse of the relative AuslanderReiten translation. This can be seen as a 2-Calabi-Yau property for $\operatorname{Sub} Q$.

There is a natural correspondence between basic injective $\Lambda$-modules $Q$ and conjugacy classes of parabolic subgroups $P$ of $G$. We show that the number of non-isomorphic indecomposable direct summands of a rigid module in $\operatorname{Sub} Q$ is at most the dimension of $P \backslash G$. A rigid module in $\operatorname{Sub} Q$ with this maximal number of summands is called complete. We prove that complete rigid modules exist in $\operatorname{Sub} Q$ by constructing explicit examples attached to certain reduced words of $w_{0}$. These modules give rise to the initial seeds from which we define the cluster algebra $\mathscr{A}$. If $X \oplus T \in \operatorname{Sub} Q$ is a basic complete rigid module with $X$ indecomposable and non-projective in $\operatorname{Sub} Q$, we show that there exists a unique indecomposable module $Y \in \operatorname{Sub} Q$ non-isomorphic to $X$ such that $Y \oplus T$ is complete rigid. The module $Y \oplus T$ is said to be obtained from $X \oplus T$ by a mutation. We can attach to any complete rigid module an integer matrix encoding the mutations with respect to all its summands which are non-projective in $\operatorname{Sub} Q$. We show that these matrices follow the FominZelevinsky matrix mutation rule. This implies, as in [15], that all cluster monomials of $\mathscr{A}$ belong to the dual semicanonical basis.

To illustrate our results, we work out in detail in Section 12 a simple but instructive example. 
We take for $G$ the group of type $D_{n}$ associated with a non-degenerate quadratic form on $\mathbb{C}^{2 n}$. The variety of isotropic lines is a smooth quadric in $\mathbb{P}^{2 n-1}(\mathbb{C})$, which can be identified with $P \backslash G$ for a maximal parabolic subgroup $P$. We describe the corresponding subcategory $\operatorname{Sub} Q$, which is of finite type with $3 n-4$ indecomposable modules, and the cluster algebras $\mathscr{A}=\mathbb{C}\left[N_{P}\right]$ and $\widetilde{\mathscr{A}}=\mathbb{C}[P \backslash G]$ which have finite cluster type $\left(A_{1}\right)^{n-2}$ in the Fomin-Zelevinsky classification [11].

In contrast, the cluster algebra structure on the coordinate ring of the Grassmann variety of isotropic subspaces of dimension $n$ in $\mathbb{C}^{2 n}$ is in general of infinite type. The only exceptions are $n=4$ and $n=5$, and they are described in detail in Section 13 .

We hope that, in general, our cluster algebra structure $\mathscr{A}$ on $\mathbb{C}[P \backslash G]$ will be helpful for studying total positivity and Poisson geometry on the partial flag variety $P \backslash G$ (see e.g. [30, 32, 20]).

After this article was submitted for publication, the preprint [7] of Buan, Iyama, Reiten and Scott appeared, which contains results similar to those of Section 8 for more general subcategories. More precisely, it is proved in [7, Chapter I, Th. 3.1] that a theory of mutation for maximal rigid modules can be developed for any extension closed functorially finite subcategory of $\bmod \Lambda$.

\section{Coordinate algebras associated to parabolic subgroups}

In this section we fix our notation for algebraic groups and flag varieties. We also recall some basic facts concerning coordinate rings of partial flag varieties and their relation with coordinate rings of unipotent radicals of parabolic subgroups.

2.1 Let $\Delta$ be a Dynkin diagram of type $A, D, E$ with vertex set $I$. We denote by $n$ the cardinality $|I|$ of $I$, and by $r$ the number of positive roots of $\Delta$.

Let $G$ be a simple simply connected complex algebraic group with Dynkin diagram $\Delta$. Let $H$ be a maximal torus of $G$, and $B, B^{-}$a pair of opposite Borel subgroups containing $H$ with unipotent radicals $N, N^{-}$.

We denote by $x_{i}(t)(i \in I, t \in \mathbb{C})$ the simple root subgroups of $N$, and by $y_{i}(t)$ the corresponding simple root subgroups of $N^{-}$.

The fundamental weights of $G$ are denoted by $\varpi_{i}(i \in I)$. For a dominant integral weight $\lambda$ let $L(\lambda)$ be the (finite-dimensional left) irreducible $G$-module with highest weight $\lambda$. The $L\left(\varpi_{i}\right)$ are called fundamental representations.

Let $g \mapsto g^{T}$ be the involutive anti-automorphism of $G$ given by

$$
x_{i}(t)^{T}=y_{i}(t), \quad h^{T}=h, \quad(i \in I, h \in H) .
$$

We denote by $L(\lambda)^{*}$ the right $G$-module obtained by twisting the action of $G$ by this anti-automorphism.

The Weyl group of $G$ is denoted by $W$ and its longest element by $w_{0}$. The Coxeter generators of $W$ are denoted by $s_{i}(i \in I)$. The length of $w \in W$ is denoted by $\ell(w)$.

The Chevalley generators of the Lie algebra $\mathfrak{g}$ of $G$ are denoted by $e_{i}, f_{i}, h_{i}(i \in I)$. Here, the $e_{i}$ 's generate the Lie algebra $\mathfrak{n}$ of $N$.

The coordinate ring $\mathbb{C}[N]$ is naturally endowed with a left action of $N$

$$
(x \cdot f)(n)=f(n x), \quad(f \in \mathbb{C}[N], x, n \in N),
$$

and a right action of $N$

$$
(f \cdot x)(n)=f(x n), \quad(f \in \mathbb{C}[N], x, n \in N) .
$$


Differentiating these two actions we get left and right actions of $\mathfrak{n}$ on $\mathbb{C}[N]$. We prefer to write $e_{i}^{\dagger}(f)$ instead of $f \cdot e_{i}$ for the right action of the Chevalley generators.

2.2 Throughout the paper we fix a non-empty subset $J$ of $I$ and we denote its complement by $K=I \backslash J$.

Let $B_{K}$ be the standard parabolic subgroup 1 of $G$ generated by $B$ and the one-parameter subgroups

$$
y_{k}(t), \quad(k \in K, t \in \mathbb{C}) .
$$

We denote by $N_{K}$ the unipotent radical of $B_{K}$. In particular, we have $B_{\emptyset}=B$ and $N_{\emptyset}=N$. On the other hand, when $K=I-\{j\}$ has $n-1$ elements, $B_{K}$ is a maximal parabolic subgroup. It is known that every parabolic subgroup of $G$ is conjugate to a unique standard parabolic subgroup $B_{K}[5$, Cor. 11.17].

Let $B_{K}^{-}$be the parabolic subgroup of $G$ generated by $B^{-}$and the one-parameter subgroups

$$
x_{k}(t), \quad(k \in K, t \in \mathbb{C}) .
$$

Then $N_{K}$ is an affine space of the same dimension as the projective variety $B_{K}^{-} \backslash G$. More precisely, the natural projection map

$$
\pi: G \rightarrow B_{K}^{-} \backslash G
$$

restricts to an embedding of $N_{K}$ into $B_{K}^{-} \backslash G$ as a dense open subset (see e.g. [5, Prop. 14.21]).

Example 2.1 Let $G=S L_{5}(\mathbb{C})$, a group of type $A_{4}$. We choose for $B$ the subgroup of upper triangular matrices. Take $J=\{2\}$ and $K=\{1,3,4\}$. Then $B_{K}^{-}$and $N_{K}$ are the subgroups of $G$ with the following block form:

$$
B_{K}^{-}=\left(\begin{array}{ccccc}
* & * & 0 & 0 & 0 \\
* & * & 0 & 0 & 0 \\
* & * & * & * & * \\
* & * & * & * & * \\
* & * & * & * & *
\end{array}\right), \quad N_{K}=\left(\begin{array}{ccccc}
1 & 0 & * & * & * \\
0 & 1 & * & * & * \\
0 & 0 & 1 & 0 & 0 \\
0 & 0 & 0 & 1 & 0 \\
0 & 0 & 0 & 0 & 1
\end{array}\right)
$$

In this case $B_{K}^{-}$is a maximal parabolic subgroup. Let $\left(u_{1}, \ldots, u_{5}\right)$ be the canonical basis of $\mathbb{C}^{5}$. We regard vectors of $\mathbb{C}^{5}$ as row vectors and we let $G$ act on the right on $\mathbb{C}^{5}$, so that the $k$ th row of the matrix $g$ is $u_{k} g$. Then $B_{K}^{-}$is the stabilizer of the 2 -space spanned by $u_{1}$ and $u_{2}$ for the induced transitive action of $G$ on the Grassmann variety of 2-planes of $\mathbb{C}^{5}$. Hence $B_{K}^{-} \backslash G$ is the Grassmannian $\operatorname{Gr}(2,5)$ of dimension 6 .

2.3 The partial flag variety $B_{K}^{-} \backslash G$ can be naturally embedded as a closed subset in the product of projective spaces

$$
\prod_{j \in J} \mathbb{P}\left(L\left(\varpi_{j}\right)^{*}\right)
$$

[26, p.123]. This is called the Plücker embedding. We denote by $\mathbb{C}\left[B_{K}^{-} \backslash G\right]$ the multi-homogeneous coordinate ring of $B_{K}^{-} \backslash G$ coming from this embedding. Let $\Pi_{J} \cong \mathbb{N}^{J}$ denote the monoid of dominant integral weights of the form

$$
\lambda=\sum_{j \in J} a_{j} \varpi_{j}, \quad\left(a_{j} \in \mathbb{N}\right) .
$$

\footnotetext{
${ }^{1}$ A more familiar notation for this parabolic subgroup would be $P_{K}$, but we keep this symbol for denoting certain projective modules over the preprojective algebra $\Lambda$.
} 
Then, $\mathbb{C}\left[B_{K}^{-} \backslash G\right]$ is a $\Pi_{J}$-graded ring with a natural $G$-module structure. The homogeneous component with multi-degree $\lambda \in \Pi_{J}$ is an irreducible $G$-module with highest weight $\lambda$. In other words, we have

$$
\mathbb{C}\left[B_{K}^{-} \backslash G\right]=\bigoplus_{\lambda \in \Pi_{J}} L(\lambda)
$$

Moreover, $\mathbb{C}\left[B_{K}^{-} \backslash G\right]$ is generated by its subspace $\bigoplus_{j \in J} L\left(\varpi_{j}\right)$.

In particular, $\mathbb{C}\left[B^{-} \backslash G\right]=\bigoplus_{\lambda \in \Pi} L(\lambda)$, where the sum is over the monoid $\Pi$ of all dominant integral weights of $G$. This is equal to the affine coordinate ring $\mathbb{C}\left[N^{-} \backslash G\right]$ of the multi-cone $N^{-} \backslash G$ over $B^{-} \backslash G$, that is, to the ring

$$
\mathbb{C}\left[N^{-} \backslash G\right]=\left\{f \in \mathbb{C}[G] \mid f(n g)=f(g), n \in N^{-}, g \in G\right\}
$$

of polynomial functions on $G$ invariant under $N^{-}$. The homogeneous component of degree $\lambda$ is the subspace consisting of all functions $f$ such that $f(h g)=h^{\lambda} f(g)$ for $h \in H$ and $g \in G$. We will identify $\mathbb{C}\left[B_{K}^{-} \backslash G\right]$ with the subalgebra of $\mathbb{C}\left[N^{-} \backslash G\right]$ generated by the homogeneous elements of degree $\varpi_{j}(j \in J)$.

Example 2.2 We continue Example 2.1, The Plücker embedding of the Grassmannian $\operatorname{Gr}(2,5)$ consists in mapping the 2-plane $V$ of $\mathbb{C}^{5}$ with basis $\left(v_{1}, v_{2}\right)$ to the line spanned by $v_{1} \wedge v_{2}$ in $\Lambda^{2} \mathbb{C}^{5}$, which is isomorphic to the right $G$-module $L\left(\varpi_{2}\right)^{*}$ (remember that $G$ acts on the right on $\mathbb{C}^{5}$ ).

This induces an embedding of $\operatorname{Gr}(2,5)$ into $\mathbb{P}\left(L\left(\varpi_{2}\right)^{*}\right)$. The homogeneous coordinate ring for this embedding is isomorphic to the subring of $\mathbb{C}[G]$ generated by the functions $g \mapsto \Delta_{i j}(g)$, where $\Delta_{i j}(g)$ denotes the $2 \times 2$ minor of $g$ taken on columns $i, j$ and on the first two rows. The $\Delta_{i j}$ are called Plücker coordinates. As a $G$-module we have

$$
\mathbb{C}[\operatorname{Gr}(2,5)]=\bigoplus_{k \in \mathbb{N}} L\left(k \varpi_{2}\right),
$$

where the degree $k$ homogeneous component $L\left(k \varpi_{2}\right)$ consists of the homogeneous polynomials of degree $k$ in the Plücker coordinates.

2.4 Some distinguished elements of degree $\varpi_{j}$ in $\mathbb{C}\left[N^{-} \backslash G\right]$ are the generalized minors

$$
\Delta_{\varpi_{j}, w\left(\varpi_{j}\right)}, \quad(w \in W),
$$

(see [9, §1.4]). The image of $N_{K}$ in $B_{K}^{-} \backslash G$ under the natural projection is the open subset defined by the non-vanishing of the minors $\Delta_{\bar{\omega}_{j}, \bar{\omega}_{j}}(j \in J)$. Therefore the affine coordinate ring of $\mathbb{C}\left[N_{K}\right]$ can be identified with the subring of degree 0 homogeneous elements in the localized ring $\mathbb{C}\left[B_{K}^{-} \backslash G\right]\left[\Delta_{\bar{\omega}_{j}, \bar{\omega}_{j}}^{-1}, j \in J\right]$. Equivalently, $\mathbb{C}\left[N_{K}\right]$ can be identified with the quotient of $\mathbb{C}\left[B_{K}^{-} \backslash G\right]$ by the ideal generated by the elements $\Delta_{\varpi_{j}, \Phi_{j}}-1(j \in J)$.

Example 2.3 We continue Example 2.1 and Example 2.2. The coordinate ring of $\mathbb{C}\left[N_{K}\right]$ is isomorphic to the ring generated by the $\Delta_{i j}$ modulo the relation $\Delta_{12}=1$. This description may seem unnecessarily complicated since $N_{K}$ is just an affine space of dimension 6 and we choose a presentation with 9 generators and the Plücker relations. But these generators are better adapted to the cluster algebra structure that we shall introduce. 
2.5 Let $\operatorname{pr}_{J}: \mathbb{C}\left[B_{K}^{-} \backslash G\right] \rightarrow \mathbb{C}\left[N_{K}\right]$ denote the projection obtained by modding out the ideal generated by the elements $\Delta_{\varpi_{j}, \bar{\varpi}_{j}}-1(j \in J)$. If $\mathbb{C}\left[B_{K}^{-} \backslash G\right]$ is identified as explained above with a subalgebra of $\mathbb{C}[G]$, this map $\operatorname{pr}_{J}$ is nothing else than restriction of functions from $G$ to $N_{K}$. The restriction of $\operatorname{pr}_{J}$ to each homogeneous piece $L(\lambda)\left(\lambda \in \Pi_{J}\right)$ of $\mathbb{C}\left[B_{K}^{-} \backslash G\right]$ is injective and gives an embedding of $L(\lambda)$ into $\mathbb{C}\left[N_{K}\right]$.

We introduce a partial ordering on $\Pi_{J}$ by declaring that $\lambda \preccurlyeq \mu$ if and only if $\mu-\lambda$ is an $\mathbb{N}$-linear combination of weights $\varpi_{j}(j \in J)$.

Lemma 2.4 Let $f \in \mathbb{C}\left[N_{K}\right]$. There is a unique homogeneous element $\tilde{f} \in \mathbb{C}\left[B_{K}^{-} \backslash G\right]$ satisfying $\operatorname{pr}_{J}(\widetilde{f})=f$ and whose multi-degree is minimal with respect to the above ordering $\preccurlyeq$.

Proof - Let us first consider the algebra $\mathbb{C}[N]$. Let $\lambda=\sum_{i} a_{i} \varpi_{i}$. It is known that the subspace $\operatorname{pr}_{I}(L(\lambda)) \subset \mathbb{C}[N]$ can be described as

$$
\operatorname{pr}_{I}(L(\lambda))=\left\{f \in \mathbb{C}[N] \mid\left(e_{i}^{\dagger}\right)^{a_{i}+1} f=0, i \in I\right\} .
$$

In particular, $\mathbb{C}\left[N_{K}\right]$ can be identified with the subalgebra

$$
\left\{f \in \mathbb{C}[N] \mid e_{k}^{\dagger} f=0, k \in K\right\} .
$$

For $\lambda=\sum_{j} a_{j} \varpi_{j} \in \Pi_{J}$, we then have

$$
\operatorname{pr}_{J}(L(\lambda))=\left\{f \in \mathbb{C}\left[N_{K}\right] \mid\left(e_{j}^{\dagger}\right)^{a_{j}+1} f=0, j \in J\right\} .
$$

Now given $f \in \mathbb{C}\left[N_{K}\right]$, we define $a_{j}(f)=\max \left\{s \mid\left(e_{j}^{\dagger}\right)^{s} f \neq 0\right\}$ and put $\lambda(f)=\sum_{j \in J} a_{j}(f) \varpi_{j}$. Then $f \in \operatorname{pr}_{J}(L(\lambda(f)))$ and $\lambda(f)$ is minimal with this property. Since the restriction of $\operatorname{pr}_{J}$ to $L(\lambda(f))$ is injective, we see that there exists a unique $\widetilde{f}$ as claimed : this is the unique element of multi-degree $\lambda(f)$ in $\mathbb{C}\left[B_{K}^{-} \backslash G\right]$ with $\operatorname{pr}_{J}(\widetilde{f})=f$.

For $f \in \mathbb{C}\left[N_{K}\right]$, let $a_{j}(f)$ and $\lambda(f)$ be as in the proof of Lemma 2.4 .

Lemma 2.5 Let $f, g \in \mathbb{C}\left[N_{K}\right]$. Then $\widetilde{(f \cdot g)}=\widetilde{f} \cdot \widetilde{g}$. If moreover, for every $j \in J$ we have $a_{j}(f+g)=$ $\max \left\{a_{j}(f), a_{j}(g)\right\}$, then

$$
\widetilde{(f+g)}=\mu \widetilde{f}+v \widetilde{g}
$$

where $\mu$ and $v$ are monomials in the variables $\Delta_{\varpi_{j}, \varpi_{j}}(j \in J)$ without common divisor.

Proof - The endomorphisms $e_{j}^{\dagger}$ are derivations of $\mathbb{C}\left[N_{K}\right]$, that is, we have

$$
e_{j}^{\dagger}(f g)=e_{j}^{\dagger}(f) g+f e_{j}^{\dagger}(g), \quad\left(f, g \in \mathbb{C}\left[N_{K}\right]\right)
$$

Therefore, by the Leibniz formula,

$$
\left(e_{j}^{\dagger}\right)^{a_{j}(f)+a_{j}(g)}(f g)=\left(e_{j}^{\dagger}\right)^{a_{j}(f)}(f)\left(e_{j}^{\dagger}\right)^{a_{j}(g)}(g) \neq 0,
$$

and $\left(e_{j}^{\dagger}\right)^{a_{j}(f)+a_{j}(g)+1}(f g)=0$. This proves the first statement. For the second statement, the additional assumption implies that there exist unique monomials $\mu$ and $v$ in the $\Delta_{\varpi_{j}, \varpi_{j}}$ such that $\mu \tilde{f}$ and $v \widetilde{g}$ have the same multi-degree as $\widetilde{(f+g)}$. Moreover $\mu$ and $v$ have no common factor. The result follows. 
Example 2.6 We continue Examples 2.1, 2.2 and 2.3, For $1 \leqslant i<j \leqslant 5$, let $D_{i j}=\operatorname{pr}_{J}\left(\Delta_{i j}\right)$. Thus, $D_{12}=1$, and for $(i, j) \neq(1,2), \widetilde{D_{i j}}=\Delta_{i j}$. It follows for example that, if $f=D_{13} D_{24}$ and $g=-D_{23} D_{14}$, then $\widetilde{f}=\Delta_{13} \Delta_{24}$ and $\widetilde{g}=-\Delta_{23} \Delta_{14}$. Now we have the Plücker relation

$$
\Delta_{13} \Delta_{24}-\Delta_{23} \Delta_{14}=\Delta_{12} \Delta_{34}
$$

This shows that $\widetilde{f+g}=\Delta_{34}$ is not of the form $\mu \widetilde{f}+v \widetilde{g}$. Here, we have $a_{2}(f)=a_{2}(g)=2$ but $a_{2}(f+g)=1$, so that the assumption $a_{2}(f+g)=\max \left\{a_{2}(f), a_{2}(g)\right\}$ is not fulfilled.

\section{The category $\operatorname{Sub} Q_{J}$}

3.1 Let $\Lambda$ be the preprojective algebra of $\Delta$ (see e.g. [31, 12]). This is a finite-dimensional selfinjective algebra over $\mathbb{C}$. An important property of $\Lambda$ is its $\operatorname{Ext}^{1}$-symmetry:

$$
\operatorname{dim} \operatorname{Ext}_{\Lambda}^{1}(M, N)=\operatorname{dim}_{E_{x t}^{1}}^{1}(N, M), \quad(M, N \in \bmod \Lambda) .
$$

In particular, $\operatorname{Ext}_{\Lambda}^{1}(M, N)=0$ if and only if $\operatorname{Ext}_{\Lambda}^{1}(N, M)=0$. In this case, we say that the modules $M$ and $N$ are orthogonal. A $\Lambda$-module $M$ is called rigid if it is orthogonal to itself, that is, if $\operatorname{Ext}_{\Lambda}^{1}(M, M)=0$.

The simple $\Lambda$-module indexed by $i \in I$ is denoted by $S_{i}$. Let $P_{i}$ be the projective cover and $Q_{i}$ the injective hull of $S_{i}$. We denote by $\mu$ the Nakayama involution of $I$, defined by $Q_{i}=P_{\mu(i)}$. (Equivalently, $\mu$ is characterized in terms of the Weyl group $W$ by $s_{\mu(i)}=w_{0} s_{i} w_{0}$.)

Let $\tau$ be the Auslander-Reiten translation of $\bmod \Lambda$, and let $\Omega$ be the syzygy functor. It is known that $\Omega$ and $\tau^{-1}$ are isomorphic as autoequivalences of the stable category $\underline{\bmod } \Lambda$ (see e.g. [14, §7.5]).

3.2 Let $Q_{J}=\bigoplus_{j \in J} Q_{j}$ and $P_{J}=\bigoplus_{j \in J} P_{j}$. We denote by $\operatorname{Sub} Q_{J}$ (resp. Fac $P_{J}$ ) the full subcategory of $\bmod \Lambda$ whose objects are isomorphic to a submodule of a direct sum of copies of $Q_{J}$ (resp. to a factor module of a direct sum of copies of $P_{J}$ ). We are going to derive cluster algebra structures on the coordinate algebras $\mathbb{C}\left[N_{K}\right]$ and $\mathbb{C}\left[B_{K}^{-} \backslash G\right]$ by constructing a mutation operation on maximal rigid modules in $\operatorname{Sub} Q_{J}$. We could alternatively use the subcategory Fac $P_{J}$, but since this would lead to the same cluster structures we shall only work with $\operatorname{Sub} Q_{J}$.

For any unexplained terminology related to subcategories, we refer the reader to the introduction of [1]. The next Proposition records some classical properties of $\operatorname{Sub} Q_{J}$ [1, Prop. 6.1].

Proposition 3.1 The subcategory $\operatorname{Sub} Q_{J}$ is closed under extensions, functorially finite, and has almost split sequences.

We shall denote by $\tau_{J}$ the relative Auslander-Reiten translation of $\operatorname{Sub} Q_{J}$. Thus for $M$ an indecomposable object of $\operatorname{Sub} Q_{J}$ which is not Ext-projective, we have $N=\tau_{J}(M)$ if and only if there is an almost split sequence in $\operatorname{Sub} Q_{J}$ of the form

$$
0 \rightarrow N \rightarrow E \rightarrow M \rightarrow 0
$$

In this situation we also write $\tau_{J}^{-1}(N)=M$. 
3.3 For each $M$ in $\bmod \Lambda$, there is a unique submodule $\theta_{J}(M)$ minimal with respect to the property that $M / \theta_{J}(M)$ is in $\operatorname{Sub} Q_{J}$. The natural projection $M \rightarrow M / \theta_{J}(M)$ is a minimal left $\operatorname{Sub} Q_{J^{-}}$ approximation of $M$ (see [25, §4]). For $i \in I$, define $L_{i}=P_{i} / \theta_{J}\left(P_{i}\right)$.

Proposition $3.2 \quad$ (i) The $L_{i}$ are the indecomposable Ext-projective and Ext-injective modules in the subcategory $\operatorname{Sub} Q_{J}$.

(ii) The direct sum of the $L_{i}$ is a minimal finite cover and a minimal finite cocover of $\operatorname{Sub} Q_{J}$.

Proof - The $L_{i}$ are the indecomposable Ext-projectives by [1, Prop. 6.3 (b)]. They are also Ext-injective because of the Ext-symmetry of $\bmod \Lambda$. Since, by [1, Prop. 6.3 (d)] the number of indecomposable Ext-injectives is the same as the number of indecomposable Ext-projectives, the $L_{i}$ are also all the indecomposable Ext-injectives. This proves (i), and (ii) follows from [1, Prop. 3.1].

3.4 We introduce the relative syzygy functor $\Omega_{J}$ of $\operatorname{Sub} Q_{J}$. For $M \in \operatorname{Sub} Q_{J}, \Omega_{J}(M)$ is defined as the kernel of the projective cover of $M$ in $\operatorname{Sub} Q_{J}$.

Lemma 3.3 For $M \in \operatorname{Sub} Q_{J}$ we have

$$
\Omega_{J}(M)=\Omega(M) / \theta_{J}(\Omega(M)) .
$$

Proof - Let $0 \rightarrow \Omega(M) \stackrel{\imath}{\rightarrow} P \stackrel{\pi}{\rightarrow} M \rightarrow 0$ be the exact sequence defining $\Omega(M)$. Since $M \in \operatorname{Sub} Q_{J}$, we have $\theta_{J}(P) \subseteq \operatorname{ker} \pi=\imath\left(\Omega(M)\right.$, hence $\theta_{J}(P)=\imath(N)$ for some $N \subseteq \Omega(M)$. The induced sequence $0 \rightarrow \Omega(M) / N \rightarrow P / \theta_{J}(P) \rightarrow M \rightarrow 0$ is exact, so $\Omega(M) / N$ is isomorphic to a submodule of $P / \theta_{J}(P) \in \operatorname{Sub} Q_{J}$, therefore $\Omega(M) / N \in \operatorname{Sub} Q_{J}$ and $\theta_{J}(\Omega(M)) \subseteq N$. Now, the induced sequence $0 \rightarrow \Omega(M) / \theta_{J}(\Omega(M)) \rightarrow P / \imath\left(\theta_{J}(\Omega(M))\right) \rightarrow M \rightarrow 0$ is also exact, and since Sub $Q_{J}$ is closed under extensions, this implies that $P / \imath\left(\theta_{J}(\Omega(M))\right) \in \operatorname{Sub} Q_{J}$, hence $\theta_{J}(P) \subseteq \imath\left(\theta_{J}(\Omega(M))\right)$, that is, $N \subseteq \theta_{J}(\Omega(M))$. Therefore the sequence $0 \rightarrow \Omega(M) / \theta_{J}(\Omega(M)) \rightarrow P / \theta_{J}(P) \rightarrow M \rightarrow 0$ is exact in $\operatorname{Sub} Q_{J}$, with middle term the projective cover of $M$ in $\operatorname{Sub} Q_{J}$, by Proposition 3.2

Proposition 3.4 For any indecomposable non-projective module $M$ in $\operatorname{Sub} Q_{J}$ we have

$$
\tau_{J}^{-1}(M)=\Omega_{J}(M)
$$

Proof - Let $0 \rightarrow M \rightarrow E \rightarrow \tau^{-1}(M) \rightarrow 0$ be an almost split sequence in $\bmod \Lambda$. Then by [1, Corollary 3.5], $\tau_{J}^{-1}(M)$ is a direct summand of $\tau^{-1}(M) / \theta_{J}\left(\tau^{-1}(M)\right)$. Now, it is known that $\tau^{-1}(M) / \theta_{J}\left(\tau^{-1}(M)\right)$ is indecomposable [3, 22], see also [25, Proposition 4.1]. Hence

$$
\tau_{J}^{-1}(M)=\tau^{-1}(M) / \theta_{J}\left(\tau^{-1}(M)\right)=\Omega(M) / \theta_{J}(\Omega(M))=\Omega_{J}(M) .
$$

To summarize, the subcategory $\operatorname{Sub} Q_{J}$ is a Frobenius category, i.e. it is an exact category with enough Ext-projectives, enough Ext-injectives, and moreover Ext-projectives and Ext-injectives coincide (see [21]). By Proposition 3.4, the corresponding stable category $\underline{\operatorname{Sub}} Q_{J}$ is a 2-CalabiYau triangulated category (see [24]). 


\section{The category $\left(\operatorname{Sub} Q_{J}\right)^{\perp}$}

A $\Lambda$-module $M$ is said to be orthogonal to $\operatorname{Sub} Q_{J}$ if $\operatorname{Ext}_{\Lambda}^{1}(M, N)=0$ for every $N \in \operatorname{Sub} Q_{J}$. We now give a useful characterization of these modules.

4.1 Let $\Delta_{K}$ be the subdiagram of $\Delta$ with vertex set $K$. This is in general a disconnected graph whose connected components are smaller Dynkin diagrams. Let $\Lambda_{K}$ be the preprojective algebra of $\Delta_{K}$. It is isomorphic to the direct product of the preprojective algebras of the connected components of $\Delta_{K}$. Although [15] only deals with preprojective algebras associated with a single Dynkin diagram (the simple case), it is easy to check that all the results extend to this more general "semisimple case". In particular, maximal rigid $\Lambda_{K}$-modules have $r_{K}$ non-isomorphic indecomposable direct summands, where $r_{K}$ denotes the number of positive roots of $\Delta_{K}$, that is, the sum of the number of positive roots of each connected component of $\Delta_{K}$.

In the sequel we shall always regard $\bmod \Lambda_{K}$ as the subcategory of $\bmod \Lambda$ whose objects are the $\Lambda$-modules supported on $K$.

Proposition 4.1 As above let $K=I \backslash J$. Then the following hold:

(i) A $\Lambda$-module $M$ with no projective indecomposable direct summand satisfies $\operatorname{Ext}_{\Lambda}^{1}(M, N)=0$ for every $N \in \operatorname{Sub} Q_{J}$ if and only if $M=\tau(U)$ with $U \in \bmod \Lambda_{K}$. In other words,

$$
\left(\operatorname{Sub} Q_{J}\right)^{\perp}=\operatorname{add}\left(\tau\left(\bmod \Lambda_{K}\right) \cup \operatorname{add} \Lambda\right) .
$$

(ii) A $\Lambda$-module $N$ with no projective indecomposable direct summand satisfies $\operatorname{Ext}_{\Lambda}^{1}(\tau(U), N)=$ 0 for every $U \in \bmod \Lambda_{K}$ if and only if $N \in \operatorname{Sub} Q_{J}$. In other words,

$$
\left(\tau\left(\bmod \Lambda_{K}\right)\right)^{\perp}=\operatorname{add}\left(\operatorname{Sub} Q_{J} \cup \operatorname{add} \Lambda\right) .
$$

Proof - (i) By [1, Prop 5.6], $M$ satisfies the above property if and only if

$$
\operatorname{Hom}_{\Lambda}\left(\tau^{-1}(M), Q_{J}\right)=0 .
$$

Now if $f: A \rightarrow Q_{J}$ is a nonzero homomorphism, then $f(A)$ is a submodule of $Q_{J}$ and its socle contains a module $S_{j}$ with $j \in J$. Therefore $A$ has a composition factor isomorphic to $S_{j}$ and $A \notin \bmod \Lambda_{K}$. Conversely, if $A$ has a composition factor of the form $S_{j}$ with $j \in J$ we can find a submodule $B$ of $A$ such that the socle of $A / B$ contains only copies of $S_{j}$. Hence $A / B$ embeds in a sum of copies of $Q_{j}$, and $\operatorname{Hom}_{\Lambda}\left(A, Q_{J}\right) \neq 0$. So we have proved that $\operatorname{Hom}_{\Lambda}\left(\tau^{-1}(M), Q_{J}\right)=0$ if and only if all the composition factors of $U=\tau^{-1}(M)$ are of the form $S_{k}$ with $k \in K$, that is, $U \in \bmod \Lambda_{K}$. This finishes the proof of $(i)$.

(ii) By part (i), if $N \in \operatorname{Sub} Q_{J}$ then $\operatorname{Ext}_{\Lambda}^{1}(\tau(U), N)=0$ for every $U \in \bmod \Lambda_{K}$. Conversely, if $N \in \bmod \Lambda$ is such that $\operatorname{Ext}_{\Lambda}^{1}(\tau(U), N)=\operatorname{Ext}_{\Lambda}^{1}(N, \tau(U))=0$ for every $U \in \bmod \Lambda_{K}$, then by taking $U=S_{k}(k \in K)$ and using the classical formula

$$
\operatorname{Ext}_{\Lambda}^{1}(A, B) \simeq \underline{\operatorname{Hom}}_{\Lambda}\left(\tau^{-1}(B), A\right)
$$

we get that $\underline{\operatorname{Hom}}_{\Lambda}\left(S_{k}, N\right)=0$. Therefore, every non-zero homomorphism from $S_{k}$ to $N$ factors through a projective-injective $\Lambda$-module, which can only be $Q_{k}$. This implies that $Q_{k}$ is a summand of $N$, contrary to the hypothesis. Hence we have that $\operatorname{Hom}_{\Lambda}\left(S_{k}, N\right)=0$, and since this holds for every $k \in K$, it follows that $N \in \operatorname{Sub} Q_{J}$.

We can now give an alternative description of the modules $L_{i}$ of Proposition 3.2 We denote by $q_{k}(k \in K)$ the indecomposable injective $\Lambda_{K}$-modules, that is, $q_{k}$ is the injective hull of $S_{k}$ in $\bmod \Lambda_{K}$. 
Proposition 4.2 Let $i \in I$. If $\mu(i)=j \in J$ then $L_{i}=Q_{j}=P_{i}$. Otherwise, if $\mu(i)=k \in K$ then $L_{i}=\tau\left(q_{k}\right)$.

Proof - Clearly, by definition of $L_{i}$, if $\mu(i)=j \in J$ then $L_{i}=Q_{j}=P_{i}$. On the other hand, for every $U \in \bmod \Lambda_{K}$ we have

$$
\operatorname{Ext}_{\Lambda}^{1}\left(\tau(U), \tau\left(q_{k}\right)\right) \simeq \operatorname{Ext}_{\Lambda}^{1}\left(U, q_{k}\right)=\operatorname{Ext}_{\Lambda_{K}}^{1}\left(U, q_{k}\right)=0, \quad(k \in K) .
$$

Hence, by Proposition 4.1(ii), we have $\tau\left(q_{k}\right) \in \operatorname{Sub} Q_{J}$. Now, by Proposition 4.1(i), $\tau\left(q_{k}\right)$ is Extorthogonal to every module $N \in \operatorname{Sub} Q_{J}$, therefore $\tau\left(q_{k}\right)$, which is indecomposable, is one of the Ext-projective modules $L_{i}$ of $\operatorname{Sub} Q_{J}$. Finally, since $\tau=\Omega^{-1}$, the head of $\tau\left(q_{k}\right)$ is $S_{i}$ with $i=\mu(k)$, so $\tau\left(q_{k}\right)=L_{i}$.

\section{The functors $\mathscr{E}_{i}$ and $\mathscr{E}_{i}^{\dagger}$}

In this section we introduce certain endo-functors $\mathscr{E}_{i}$ and $\mathscr{E}_{i}^{\dagger}$ of $\bmod \Lambda$ which we use to construct rigid $\Lambda$-modules. These functors should be seen as the lifts to $\bmod \Lambda$ of the maps $e_{i}^{\max }$ and $\left(e_{i}^{\dagger}\right)^{\max }$ from $\mathbb{C}[N]$ to $\mathbb{C}[N]$ defined by

$$
e_{i}^{\max } f=\left(e_{i}^{k} / k !\right) f \quad \text { where } \quad k=\max \left\{j \mid e_{i}^{j} f \neq 0\right\},
$$

and

$$
\left(e_{i}^{\dagger}\right)^{\max } f=\left(e_{i}^{\dagger l} / l !\right) f \quad \text { where } \quad l=\max \left\{j \mid e_{i}^{\dagger j} f \neq 0\right\} .
$$

5.1 For $M \in \bmod \Lambda$ and $i \in I$, let $m_{i}^{\dagger}(M)$ denote the multiplicity of $S_{i}$ in the socle of $M$. We define an endo-functor $\mathscr{E}_{i}^{\dagger}$ of $\bmod \Lambda$ as follows. For an object $M \in \bmod \Lambda$ we put

$$
\mathscr{E}_{i}^{\dagger}(M)=M / S_{i}^{\oplus m_{i}^{\dagger}(M)}
$$

So we get a short exact sequence

$$
0 \rightarrow S_{i}^{\oplus m_{i}^{\dagger}(M)} \rightarrow M \rightarrow \mathscr{E}_{i}^{\dagger}(M) \rightarrow 0
$$

If $f: M \rightarrow N$ is a homomorphism, we can compose it with the natural projection $N \rightarrow \mathscr{E}_{i}^{\dagger}(N)$ to obtain $\tilde{f}: M \rightarrow \mathscr{E}_{i}^{\dagger}(N)$. The $S_{i}$-isotypic component of the socle of $M$ is mapped to 0 by $\widetilde{f}$, hence $\widetilde{f}$ induces a homomorphism $\mathscr{E}_{i}^{\dagger}(f): \mathscr{E}_{i}^{\dagger}(M) \rightarrow \mathscr{E}_{i}^{\dagger}(N)$. Clearly, $\mathscr{E}_{i}^{\dagger}$ is an additive functor.

Proposition 5.1 The functors $\mathscr{E}_{i}^{\dagger}(i \in I)$ satisfy the following relations:

(i) $\mathscr{E}_{i}^{\dagger} \mathscr{E}_{i}^{\dagger}=\mathscr{E}_{i}^{\dagger}$

(ii) $\mathscr{E}_{i}^{\dagger} \mathscr{E}_{j}^{\dagger}=\mathscr{E}_{j}^{\dagger} \mathscr{E}_{i}^{\dagger}$ if $i$ and $j$ are not connected by an edge in $\Delta$.

(iii) $\mathscr{E}_{i}^{\dagger} \mathscr{E}_{j}^{\dagger} \mathscr{E}_{i}^{\dagger}=\mathscr{E}_{j}^{\dagger} \mathscr{E}_{i}^{\dagger} \mathscr{E}_{j}^{\dagger}$ if $i$ and $j$ are connected by an edge in $\Delta$. 
Proof - Claim (i) is evident. Next consider the largest submodule $M_{i, j}$ of $M$ whose composition factors are all isomorphic to $S_{i}$ or $S_{j}$. If $i$ and $j$ are not connected by an edge in $\Delta$ then $\operatorname{Ext}_{\Lambda}^{1}\left(S_{i}, S_{j}\right)=\operatorname{Ext}_{\Lambda}^{1}\left(S_{j}, S_{i}\right)=0$, hence $M_{i, j}$ is semisimple, and one clearly has

$$
\mathscr{E}_{i}^{\dagger} \mathscr{E}_{j}^{\dagger}(M)=\mathscr{E}_{j}^{\dagger} \mathscr{E}_{i}^{\dagger}(M)=M / M_{i, j}
$$

If $i$ and $j$ are connected by an edge in $\Delta$, an elementary calculation in type $A_{2}$ shows that

$$
\mathscr{E}_{i}^{\dagger} \mathscr{E}_{j}^{\dagger} \mathscr{E}_{i}^{\dagger}\left(M_{i, j}\right)=\mathscr{E}_{j}^{\dagger} \mathscr{E}_{i}^{\dagger} \mathscr{E}_{j}^{\dagger}\left(M_{i, j}\right)=0
$$

It follows that

$$
\mathscr{E}_{i}^{\dagger} \mathscr{E}_{j}^{\dagger} \mathscr{E}_{i}^{\dagger}(M)=\mathscr{E}_{j}^{\dagger} \mathscr{E}_{i}^{\dagger} \mathscr{E}_{j}^{\dagger}(M)=M / M_{i, j}
$$

Let $\left(i_{1}, \ldots, i_{k}\right)$ be a reduced word for $w \in W$. Proposition 5.1 implies that the functor $\mathscr{E}_{i_{1}}^{\dagger} \cdots \mathscr{E}_{i_{k}}^{\dagger}$ does not depend on the choice of the reduced word. We shall denote it by $\mathscr{E}_{w}^{\dagger}$.

5.2 For $M \in \bmod \Lambda$ let $m_{i}(M)$ be the multiplicity of $S_{i}$ in the top of $M$. We define an endo-functor $\mathscr{E}_{i}$ of $\bmod \Lambda$ as follows. For an object $M \in \bmod \Lambda$ we define $\mathscr{E}_{i}(M)$ as the kernel of the surjection

$$
M \rightarrow S_{i}^{\oplus m_{i}(M)}
$$

If $f: M \rightarrow N$ is a homomorphism, $f\left(\mathscr{E}_{i}(M)\right)$ is contained in $\mathscr{E}_{i}(N)$, and we define

$$
\mathscr{E}_{i}(f): \mathscr{E}_{i}(M) \rightarrow \mathscr{E}_{i}(N)
$$

as the restriction of $f$ to $\mathscr{E}_{i}(M)$. Clearly, $\mathscr{E}_{i}$ is a functor. Alternatively, we could define $\mathscr{E}_{i}$ as the composition of functors

$$
\mathscr{E}_{i}=S \mathscr{E}_{i}^{\dagger} S
$$

where $S$ is the self-duality of $\bmod \Lambda$ introduced in [14, §1.7]. This shows immediately that the functors $\mathscr{E}_{i}$ satisfy analogues of Proposition 5.1. In particular, we have for every $w \in W$ a welldefined functor $\mathscr{E}_{w}$.

Proposition 5.2 If $M$ is rigid then $\mathscr{E}_{i}(M)$ and $\mathscr{E}_{i}^{\dagger}(M)$ are rigid.

Proof - Let $M$ be a rigid module with dimension vector $\beta$. We use the geometric characterization of rigid $\Lambda$-modules given in [15, Cor. 3.15], namely, $M$ is rigid if and only if its orbit $\mathscr{O}_{M}$ in the variety $\Lambda_{\beta}$ of $\Lambda$-modules of dimension vector $\beta$ is open.

Let $p=m_{i}(M)$ and $\beta^{\prime}=\beta-p \alpha_{i}$. In [28, $\left.\S 12.2\right]$, Lusztig considers, for $q \in \mathbb{N}$, the subvariety $\Lambda_{\beta, i, q}$ of $\Lambda_{\beta}$ consisting of all $\Lambda$-modules $N$ with $m_{i}(N)=q$. He introduces the variety $Y$ of triples $(t, s, r)$ where $t \in \Lambda_{\beta^{\prime}, i, 0}, s \in \Lambda_{\beta, i, p}$ and $r: t \rightarrow s$ is an injective homomorphism of $\Lambda$-modules. Let $p_{1}: Y \rightarrow \Lambda_{\beta^{\prime}, i, 0}$ and $p_{2}: Y \rightarrow \Lambda_{\beta, i, p}$ denote the natural projections. It is easy to check that $p_{1} p_{2}^{-1}\left(\mathscr{O}_{N}\right)=\mathscr{O}_{\mathscr{E}_{i}(N)}$, the orbit of $\mathscr{E}_{i}(N)$ in $\Lambda_{\beta^{\prime}}$. By [28, Lemma 12.5], $p_{2}$ is a principal $G_{\beta^{\prime}}$ bundle, and the map $(t, s, r) \mapsto(t, r)$ is a locally trivial fibration $p_{1}^{\prime}: Y \rightarrow \Lambda_{\beta^{\prime}, i, 0} \times J_{0}$ with fibre isomorphic to $\mathbb{C}^{m}$ for a certain $m \in \mathbb{N}$. Here, $J_{0}$ stands for the set of all injective graded linear maps from $V_{\beta^{\prime}}$ to $V_{\beta}$. It follows that if $\mathscr{O}_{M}$ is open in $\Lambda_{\beta}$ then $p_{1}^{\prime} p_{2}^{-1}\left(\mathscr{O}_{M}\right)$ is open in $\Lambda_{\beta^{\prime}, i, 0} \times J_{0}$, and $\mathscr{O}_{\mathscr{E} i}(M)$ is open in $\Lambda_{\beta^{\prime}, i, 0}$. Since $\Lambda_{\beta^{\prime}, i, 0}$ has the same pure dimension as $\Lambda_{\beta^{\prime}}$ [28, Th. 12.3], we 
conclude that $\mathscr{O}_{\mathscr{E}_{i}(M)}$ is open in $\Lambda_{\beta^{\prime}}$. This proves the claim for $\mathscr{E}_{i}(M)$. The result for $\mathscr{E}_{i}^{\dagger}(M)$ then follows from (1).

If $M$ is a rigid $\Lambda$-module with dimension vector $\beta$ then $\overline{\mathscr{O}_{M}}$ is an irreducible component of $\Lambda_{\beta}$. So the functors $\mathscr{E}_{i}$ yield maps which associate to an irreducible component containing a rigid module, other irreducible components, also containing a rigid module. By [23], these maps correspond to certain arrows of Lusztig's coloured graph for the enveloping algebra $U(\mathfrak{n})$ of Dynkin type $\Delta$ (see [29, $\S 14.4 .7, \S 14.4 .15])$ or, equivalently, to certain paths in Kashiwara's crystal graph of $U(\mathfrak{n})$.

5.3 The functors $\mathscr{E}_{i}$ and $\mathscr{E}_{i}^{\dagger}$ can be used to construct rigid $\Lambda$-modules. Start with $Q=\oplus_{i \in I} Q_{i}$. This is a projective-injective module, hence a rigid module. Let $\mathbf{i}=\left(i_{1}, \ldots, i_{r}\right)$ be a reduced word for $w_{0}$. We define a rigid module $T_{\mathbf{i}}^{\dagger}$ by induction as follows. Put ${ }^{\dagger} T^{(r+1)}=Q$, and

$$
{ }^{\dagger} T^{(k)}=\mathscr{E}_{i_{k}}^{\dagger}\left({ }^{\dagger} T^{(k+1)}\right) \oplus Q_{i_{k}}, \quad(1 \leqslant k \leqslant r) .
$$

Then define $T_{\mathbf{i}}^{\dagger}:={ }^{\dagger} T^{(1)}$.

Example 5.3 In type $A_{3}$, let $\mathbf{i}=(2,1,3,2,1,3)$. We have

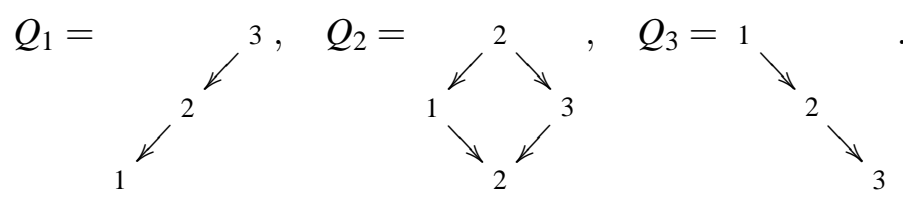

Here the graphs display the structure of the injective $\Lambda$-modules. Thus, $Q_{1}$ is a uniserial module with socle $S_{1}$, top $S_{3}$ and middle layer $S_{2}$. (In the sequel we shall frequently use graphs of this type to represent examples of $\Lambda$-modules.) Now,

$$
{ }^{\dagger} T^{(6)}=\mathscr{E}_{3}^{\dagger}(Q) \oplus Q_{3}=\mathscr{E}_{3}^{\dagger}\left(Q_{3}\right) \oplus Q=1 \searrow_{2} \oplus Q .
$$

Next,

$$
{ }^{\dagger} T^{(5)}=\mathscr{E}_{1}^{\dagger}\left({ }^{\dagger} T^{(6)}\right) \oplus Q_{1}=1 \searrow_{2} \oplus \swarrow_{2} \swarrow^{3 \oplus Q .}
$$

Similarly we get

$$
\begin{aligned}
& { }^{\dagger} T^{(4)}=1 \oplus 3 \oplus{ }^{\dagger} T^{(2)}=1 \searrow_{2} \oplus 2 \oplus \oplus \downarrow_{3} \oplus Q, \quad{ }^{\dagger} T^{(3)}=1 \oplus{ }^{3} \oplus Q, \quad{ }^{\dagger} T^{(1)}=1 \oplus 3 \oplus{ }^{3} \oplus{ }^{2} \oplus \searrow_{2} \oplus Q, \\
& { }^{2} \searrow_{3} \oplus Q=T_{\mathbf{i}}^{\dagger} .
\end{aligned}
$$

5.4 Similarly we can define a rigid module $T_{\mathbf{i}}$ inductively as follows. Put $T^{(r+1)}=Q$, and

$$
T^{(k)}=\mathscr{E}_{i_{k}}\left(T^{(k+1)}\right) \oplus P_{i_{k}}, \quad(1 \leqslant k \leqslant r)
$$

Then define

$$
T_{\mathbf{i}}:=T^{(1)} .
$$

In fact, we have $T_{\mathbf{i}}=S T_{\mathbf{i}}^{\dagger}$. In particular, $T_{\mathbf{i}}$ and $T_{\mathbf{i}}^{\dagger}$ have the same number of indecomposable direct summands, and their endomorphism algebras have opposite Gabriel quivers. 
5.5 Let $W_{K}$ be the subgroup of $W$ generated by the $s_{k}(k \in K)$. Let $w_{0}^{K}$ denote the longest element of $W_{K}$. We have $\ell\left(w_{0}^{K}\right)=r_{K}$.

Proposition 5.4 For any $\Lambda$-module $M$, we have

$$
\mathscr{E}_{w_{0}^{K}}^{\dagger}(M)=M / \theta_{J}(M)
$$

Proof - It is easily checked that for every $k \in K, \mathscr{E}_{w_{0}^{K}}^{\dagger}\left(q_{k}\right)=0$. This implies that for every $N \in \bmod \Lambda_{K}$ we have $\mathscr{E}_{w_{0}^{\dagger}}^{\dagger}(N)=0$. Therefore, if $N$ is the largest submodule of $M$ contained in $\bmod \Lambda_{K}$, we have $\mathscr{E}_{w_{0}^{K}}^{\dagger}(M)=M / N$, and the result follows.

Proposition 5.4 shows that the functor $\mathscr{E}_{w_{0}^{K}}^{\dagger}: \bmod \Lambda \rightarrow \operatorname{Sub} Q_{J}$ is left adjoint to the inclusion functor $l_{J}: \operatorname{Sub} Q_{J} \rightarrow \bmod \Lambda$ (see e.g. [2, $\{2$, p.17-18]). In other words, there are isomorphisms

$$
\operatorname{Hom}_{\Lambda}(M, N) \stackrel{\sim}{\rightarrow} \operatorname{Hom}_{\operatorname{Sub} Q_{J}}\left(\mathscr{E}_{w_{0}^{K}}^{\dagger}(M), N\right)
$$

functorial in $M \in \bmod \Lambda$ and $N \in \operatorname{Sub} Q_{J}$.

In particular, taking $K=\{k\}$, we see that $\mathscr{E}_{k}^{\dagger}$ is left adjoint to the inclusion functor $l_{I-\{k\}}$. Similarly, $\mathscr{E}_{k}$ is right adjoint to the inclusion functor of the subcategory Fac $P_{I-\{k\}}$.

\section{Generalized minors and maximal rigid modules}

6.1 For $i \in I$ and $u, v \in W$, let $\Delta_{u\left(\varpi_{i}\right), v\left(\varpi_{i}\right)}$ denote the generalized minor introduced by Fomin and Zelevinsky $[9, \S 1.4]$. This is a regular function on $G$. We shall mainly work with the restriction of this function to $N$, that we shall denote by $D_{u\left(\varpi_{i}\right), v\left(\varpi_{i}\right)}$. It is easy to see that $D_{u\left(\varpi_{i}\right), v\left(\varpi_{i}\right)}=0$ unless $u\left(\varpi_{i}\right)$ is less or equal to $v\left(\varpi_{i}\right)$ in the Bruhat order, and that $D_{u\left(\varpi_{i}\right), u\left(\varpi_{i}\right)}=1$ for every $i \in I$ and $u \in W($ see $[4, \S 2.3])$.

6.2 In [15, §9] we have associated to every $M \in \bmod \Lambda$ a regular function $\varphi_{M} \in \mathbb{C}[N]$, encoding the Euler characteristics of the varieties of composition series of $M$. In particular, one has

$$
\varphi_{Q_{i}}=D_{\bar{\varpi}_{i}, w_{0}\left(\varpi_{i}\right)}, \quad(i \in I) .
$$

More generally, it follows from [14, Lemma 5.4] that for $u, v \in W$ we have

$$
\varphi_{\mathscr{E}_{u}^{\dagger} \mathscr{E}_{v} Q_{i}}=D_{u\left(\varpi_{i}\right), v w_{0}\left(\varpi_{i}\right)} .
$$

6.3 To a reduced word $\mathbf{i}=\left(i_{1}, \ldots, i_{r}\right)$ of $w_{0}$, Fomin and Zelevinsky have attached a sequence of minors $\Delta(k, \mathbf{i})$ defined as follows (see [14, §5.3]). Here $k$ varies over $[-n,-1] \cup[1, r]$,

$$
\Delta(-j, \mathbf{i})=\Delta_{\varpi_{j}, w_{0}\left(\varpi_{j}\right)}, \quad(j \in[1, n]),
$$

and

$$
\Delta(k, \mathbf{i})=\Delta_{\bar{\omega}_{i_{k}}, v_{>k}\left(\varpi_{i_{k}}\right)}, \quad(k \in[1, r]),
$$

where $v_{>k}=s_{i_{r}} s_{i_{r-1}} \cdots s_{i_{k+1}}$. We shall denote by $D(k, \mathbf{i})$ the restriction of $\Delta(k, \mathbf{i})$ to $N$. There are $n$ indices $l_{j}(1 \leqslant j \leqslant n)$ in $[1, r]$ such that $D\left(l_{j}, \mathbf{i}\right)=D_{\varpi_{j}, \varpi_{j}}=1$. The remaining $r-n$ indices in $[1, r]$ are called $\mathbf{i}$-exchangeable. The subset of $[1, r]$ consisting of these $\mathbf{i}$-exchangeable indices is denoted by $e(\mathbf{i})$. The functions $D(k, \mathbf{i})(k \in[-n,-1] \cup e(\mathbf{i}))$ form one of the initial clusters for the cluster algebra $\mathbb{C}[N][4,14]$. 
Proposition 6.1 Let $\mathbf{i}=\left(i_{1}, \ldots, i_{r}\right)$ be a reduced word for $w_{0}$. Then the following hold:

(i) $T_{\mathbf{i}}$ is a basic complete rigid $\Lambda$-module.

(ii) We can denote the $r$ indecomposable direct summands of $T_{\mathbf{i}}$ by $T_{k}$ in such a way that

$$
\varphi_{T_{k}}=D(k, \mu(\mathbf{i})), \quad(k \in[-n,-1] \cup e(\mu(\mathbf{i}))),
$$

where $\mu(\mathbf{i})=\left(\mu\left(i_{1}\right), \ldots, \mu\left(i_{r}\right)\right)$ is the image of $\mathbf{i}$ under the Nakayama involution (see 3.1 ).

Proof - One has $\mathscr{E}_{i}\left(P_{j}\right)=P_{j}$ if $j \neq i$. Moreover, since $P_{i}$ has a simple socle, all its submodules are indecomposable. It then follows from the inductive definition of $T_{\mathbf{i}}$ that the indecomposable direct summands of $T_{\mathbf{i}}$ are the projective modules $P_{i}$ and all the nonzero modules of the form

$$
M_{k}=\mathscr{E}_{i_{1}} \mathscr{E}_{i_{2}} \cdots \mathscr{E}_{i_{k}}\left(P_{i_{k}}\right), \quad(1 \leqslant k \leqslant r) .
$$

Using Equation (3) and the fact that $P_{i}=Q_{\mu(i)}$, we thus get immediately

$$
\varphi_{M_{k}}=D_{\varpi_{\mu\left(i_{k}\right)}, u_{\leqslant k} w_{0}\left(\Phi_{\mu\left(i_{k}\right)}\right)},
$$

where $u_{\leqslant k}=s_{i_{1}} \cdots s_{i_{k}}$. Now,

$$
u_{\leqslant k} w_{0}=w_{0} s_{i_{r}} \cdots s_{i_{k+1}} w_{0}=s_{\mu\left(i_{r}\right)} \cdots s_{\mu\left(i_{k+1}\right)},
$$

and it follows that $\varphi_{M_{k}}=D(k, \mu(\mathbf{i}))$. Note that the $n$ indices $k \in[1, r]$ which are not $\mu(\mathbf{i})$-exchangeable give $\varphi_{M_{k}}=1$, hence the corresponding modules $M_{k}$ are zero. Therefore, taking into account the $n$ modules $Q_{i}$, we see that $T_{\mathbf{i}}$ has exactly $r$ pairwise non-isomorphic indecomposable direct summands and projects under the map $M \mapsto \varphi_{M}$ to the product of cluster variables

$$
D(k, \mu(\mathbf{i})), \quad(k \in[-n,-1] \cup e(\mu(\mathbf{i}))) .
$$

Proposition 6.1 gives a representation-theoretic construction of all the initial clusters of $\mathbb{C}[N]$. Note that this description is different from that of [14] which was only valid for the reduced words i adapted to an orientation of the Dynkin diagram. It is known (see [4, Remark 2.14]) that all these clusters are related to each other by mutation. Therefore, using [14] and [15] we know that the exchange matrix of the cluster

$$
\{D(k, \mu(\mathbf{i})) \mid k \in[-n,-1] \cup e(\mu(\mathbf{i}))\}
$$

coincides with the exchange matrix of the rigid module $T_{\mathbf{i}}$. Hence, using [4], we have an easy combinatorial rule to determine the exchange matrix of $T_{\mathbf{i}}$ for every $\mathbf{i}$, or in other words the Gabriel quiver of its endomorphism algebra.

6.4 For $u, v \in W$ and $i \in I$ we have

$$
S\left(\mathscr{E}_{u}^{\dagger} \mathscr{E}_{v} Q_{i}\right)=\mathscr{E}_{u} \mathscr{E}_{v}^{\dagger} P_{i}=\mathscr{E}_{v}^{\dagger} \mathscr{E}_{u} Q_{\mu(i)}
$$

It follows that

$$
\varphi_{S\left(\mathscr{E}_{u}^{\dagger} \mathscr{E}_{v} Q_{i}\right)}=D_{v\left(\varpi_{\mu(i)}\right), u w_{0}\left(\varpi_{\mu(i)}\right)} .
$$

This, together with Proposition 6.1, implies that 
Corollary 6.2 The rigid module $T_{\mathbf{i}}^{\dagger}$ is basic and complete in $\bmod \Lambda$. Its $r-n$ non-projective indecomposable direct summands $T_{k}^{\dagger}=S T_{k}$ satisfy

$$
\varphi_{T_{k}^{\dagger}}=D_{u_{\leqslant k}\left(\varpi_{i_{k}}\right), w_{0}\left(\varpi_{i_{k}}\right)}, \quad(k \in e(\mathbf{i})),
$$

where $u_{\leqslant k}=s_{i_{1}} \cdots s_{i_{k}}$.

Example 6.3 We continue Example 5.3 We have

$$
\begin{aligned}
& D_{u_{\leqslant 6}\left(\varpi_{3}\right), w_{0}\left(\varpi_{3}\right)}=1, \quad D_{u_{\leqslant 5}\left(\varpi_{1}\right), w_{0}\left(\varpi_{1}\right)}=1, \quad D_{u_{\leqslant 4}\left(\Phi_{2}\right), w_{0}\left(\varpi_{2}\right)}=1, \\
& D_{u_{\leqslant 3}\left(\varpi_{3}\right), w_{0}\left(\varpi_{3}\right)}=D_{s_{2} s_{1} s_{3}\left(\varpi_{3}\right), w_{0}\left(\varpi_{3}\right)}=\varphi_{S_{1}}, \\
& D_{u_{\leqslant 2}\left(\varpi_{1}\right), w_{0}\left(\varpi_{1}\right)}=D_{S_{2} s_{1}\left(\varpi_{1}\right), w_{0}\left(\varpi_{1}\right)}=\varphi_{S_{3}}, \\
& D_{u \leqslant 1}\left(\Phi_{2}\right), w_{0}\left(\varpi_{2}\right)=D_{s_{2}\left(\Phi_{2}\right), w_{0}\left(\Phi_{2}\right)}=\varphi \swarrow_{1}^{2} \searrow_{3} .
\end{aligned}
$$

\section{Complete rigid modules in $\operatorname{Sub} Q_{J}$}

7.1 For a $\Lambda$-module $T$, we denote by $\Sigma(T)$ the number of non-isomorphic indecomposable direct summands of $T$.

Proposition 7.1 Let $T$ be a rigid module in $\operatorname{Sub} Q_{J}$. We have $\Sigma(T) \leqslant r-r_{K}$.

Proof - We may assume without loss of generality that $T$ is basic. Let $T^{\prime}$ be a basic rigid $\Lambda_{K}$-module without projective summands. Then by [19] we know that $\Sigma\left(T^{\prime}\right) \leqslant r_{K}-|K|$, and by [14] we can assume that this upper bound is achieved, that is, we can assume that $T^{\prime}$ has $r_{K}-|K|$ indecomposable summands. It follows that $\tau\left(T^{\prime}\right)$ is a basic rigid $\Lambda$-module with $r_{K}-|K|$ indecomposable summands. By Proposition 4.1, no summand of $\tau\left(T^{\prime}\right)$ belongs to $\operatorname{Sub} Q_{J}$, since such a summand would have to be the $\tau$-translate of a projective $\Lambda_{K}$-module. Therefore the $\Lambda$ module $T^{\prime \prime}=T \oplus \tau\left(T^{\prime}\right) \oplus Q_{K}$ is basic and rigid. Here we use that for $k \in K$, the injective module $Q_{k}$ is not in $\operatorname{Sub} Q_{J}$. By [19] we have $\Sigma\left(T^{\prime \prime}\right) \leqslant r$, hence

$$
\Sigma(T)+\left(r_{K}-|K|\right)+|K|=\Sigma(T)+r_{K} \leqslant r
$$

Note that $r-r_{K}=\operatorname{dim} N_{K}=\operatorname{dim} B_{K}^{-} \backslash G$.

Definition 7.2 A rigid module $T$ in $\operatorname{Sub} Q_{J}$ is called complete if $\Sigma(T)=r-r_{K}$. 
7.2 We can write $w_{0}=w_{0}^{K} v_{K}$ with $\ell\left(w_{0}^{K}\right)+\ell\left(v_{K}\right)=\ell\left(w_{0}\right)$. Therefore there exist reduced words i for $w_{0}$ starting with a factor $\left(i_{1}, \ldots, i_{r_{K}}\right)$ which is a reduced word for $w_{0}^{K}$.

Proposition 7.3 Let $\mathbf{i}=\left(i_{1}, \ldots, i_{r}\right)$ be a reduced word for $w_{0}$ such that $\left(i_{1}, \ldots, i_{r_{K}}\right)$ is a reduced word for $w_{0}^{K}$. Then $T_{\mathbf{i}}^{\dagger}$ has $r-r_{K}$ non-isomorphic indecomposable direct summands in $\operatorname{Sub} Q_{J}$.

Proof - The indecomposable direct summands of $T_{\mathbf{i}}^{\dagger}$ are the $Q_{i}(i \in I)$ and the modules

$$
M_{j}^{\dagger}=\mathscr{E}_{i_{1}}^{\dagger} \mathscr{E}_{i_{2}}^{\dagger} \cdots \mathscr{E}_{i_{j}}^{\dagger}\left(Q_{i_{j}}\right), \quad(j \in e(\mathbf{i}))
$$

By Corollary 6.2 these $r$ summands are pairwise non-isomorphic. For $j>r_{K}$, by Proposition 5.4 we have $M_{j}^{\dagger} \in \operatorname{Sub} Q_{J}$. Taking into account the $n$ non-exchangeable elements, we have $r-n-r_{K}$ such modules. Moreover, again by Proposition 5.4 we have

$$
N_{k}^{\dagger}:=\mathscr{E}_{i_{1}}^{\dagger} \mathscr{E}_{i_{2}}^{\dagger} \cdots \mathscr{E}_{i_{r_{K}}^{\dagger}}^{\dagger}\left(Q_{k}\right)=\mathscr{E}_{w_{0}^{K}}^{\dagger} Q_{k} \in \operatorname{Sub} Q_{J}, \quad(k \in K)
$$

Let $s_{k}=\max \left\{s \leqslant r_{K} \mid i_{s}=k\right\}$. Then $M_{s_{k}}^{\dagger}=N_{k}^{\dagger}$ belongs to Sub $Q_{J}$. Finally, since for $j \in J$ we have obviously $Q_{j} \in \operatorname{Sub} Q_{J}$, we have found $r-n-r_{K}+|K|+|J|=r-r_{K}$ summands of $T_{\mathbf{i}}^{\dagger}$ in $\operatorname{Sub} Q_{J}$.

Proposition 7.3 shows that there exist complete rigid modules in $\operatorname{Sub} Q_{J}$, and gives a recipe to construct some of them. Note that the modules $L_{i}(i \in I)$ occur as direct summands of every complete rigid module $T$ in $\operatorname{Sub} Q_{J}$. This follows immediately from the fact that the $L_{i}$ are Extprojective and Ext-injective in $\operatorname{Sub} Q_{J}$.

7.3 Proposition 7.3 is based on properties of the functor $\mathscr{E}_{w_{0}^{K}}^{\dagger}$. A related result is given by the next proposition. Following Iyama, we call a $\Lambda$-module $T$ maximal 1-orthogonal in $\operatorname{Sub} Q_{J}$ if

$$
\operatorname{add} T=\left\{M \in \operatorname{Sub} Q_{J} \mid \operatorname{Ext}_{\Lambda}^{1}(T, M)=0\right\} .
$$

Clearly a maximal 1-orthogonal module $T$ is maximal rigid in $\operatorname{Sub} Q_{J}$, that is, it is rigid, and if $X \in \operatorname{Sub} Q_{J}$ is such that $T \oplus X$ is rigid then $X \in \operatorname{add} T$.

Proposition 7.4 Let $T$ be a maximal rigid module in $\bmod \Lambda$. Then $\mathscr{E}_{w_{0}^{K}}^{\dagger}(T)$ is maximal 1-orthogonal in $\operatorname{Sub} Q_{J}$.

Proof - By Proposition 5.2, $\mathscr{E}_{w_{0}^{K}}^{\dagger}(T)$ is rigid, so if $M \in \operatorname{add} \mathscr{E}_{w_{0}^{K}}^{\dagger}(T)$ then

$$
\operatorname{Ext}_{\Lambda}^{1}\left(\mathscr{E}_{w_{0}^{K}}^{\dagger}(T), M\right)=0
$$

Conversely, let us assume that $M \in \operatorname{Sub} Q_{J}$ is such that $\operatorname{Ext}_{\Lambda}^{1}\left(\mathscr{E}_{w_{0}^{K}}^{\dagger}(T), M\right)=0$.

Let $f: T^{\prime} \rightarrow M$ be a minimal right add $T$-approximation. Since $\Lambda \in \operatorname{add} T, f$ is surjective and putting $T^{\prime \prime}=\operatorname{ker} f$ we get an exact sequence

$$
0 \rightarrow T^{\prime \prime} \rightarrow T^{\prime} \rightarrow M \rightarrow 0
$$

By Wakamatsu's lemma, it follows that $\operatorname{Ext}_{\Lambda}^{1}\left(T, T^{\prime \prime}\right)=0$ (see [2, Lemma 3.1]). By [15, Th. 6.4], we know that $T$ is maximal 1 -orthogonal in $\bmod \Lambda$, hence $T^{\prime \prime} \in \operatorname{add} T$. 
Now, since $M \in \operatorname{Sub} Q_{J}$ we have $\theta_{J}(M)=0$ and $\theta_{J}\left(T^{\prime \prime}\right) \simeq \theta_{J}\left(T^{\prime}\right)$. Therefore we get an exact sequence

$$
0 \rightarrow \mathscr{E}_{w_{0}^{K}}^{\dagger}\left(T^{\prime \prime}\right) \rightarrow \mathscr{E}_{w_{0}^{K}}^{\dagger}\left(T^{\prime}\right) \rightarrow M \rightarrow 0
$$

But $\mathscr{E}_{w_{0}^{K}}^{\dagger}\left(T^{\prime \prime}\right) \in \operatorname{add} \mathscr{E}_{w_{0}^{K}}^{\dagger}(T)$, so by assumption

$$
\operatorname{Ext}_{\Lambda}^{1}\left(\mathscr{E}_{w_{0}^{K}}^{\dagger}\left(T^{\prime \prime}\right), M\right)=\operatorname{Ext}_{\Lambda}^{1}\left(M, \mathscr{E}_{w_{0}^{K}}^{\dagger}\left(T^{\prime \prime}\right)\right)=0
$$

and the sequence splits. Hence $M$ is a direct summand of $\mathscr{E}_{w_{0}^{K}}^{\dagger}\left(T^{\prime}\right)$ and $M \in \operatorname{add} \mathscr{E}_{w_{0}^{K}}^{\dagger}(T)$, as required.

7.4 It follows from [17, Cor. 8.7] that every maximal rigid module in $\operatorname{Sub} Q_{J}$ is complete. Proposition 7.4 then implies that for every maximal rigid module $T \in \bmod \Lambda$, the module $\mathscr{E}_{w_{0}^{\dagger}}^{\dagger}(T)$ is complete rigid in $\operatorname{Sub} Q_{J}$. We will not make use of this result in the sequel.

\section{Mutations in $\operatorname{Sub} Q_{J}$}

8.1 Let $X \oplus T$ be a basic complete rigid module in $\operatorname{Sub} Q_{J}$ with $X$ indecomposable and not Ext-projective. Let $V$ be a basic rigid module in $\bmod \Lambda_{K}$ without projective summand, and let us assume that $\Sigma(V)=r_{K}-|K|$. Then, by Proposition 4.1 $X \oplus T \oplus \tau(V) \oplus Q_{K}$ is a basic maximal rigid module in $\bmod \Lambda$.

Let $f: T^{\prime} \rightarrow X$ be a minimal right add $(T)$-approximation. Since $T$ contains as a summand the module $L=\oplus_{j} L_{j}$, which is a cover of $\operatorname{Sub} Q_{J}$, the map $f$ is surjective. Let $Y$ be the kernel of $f$, so that we have a short exact sequence

$$
0 \rightarrow Y \stackrel{g}{\rightarrow} T^{\prime} \stackrel{f}{\rightarrow} X \rightarrow 0
$$

where $g$ denotes inclusion. Since $T^{\prime}$ belongs to $\operatorname{Sub} Q_{J}$ which is closed under taking submodules, $Y$ also belongs to $\operatorname{Sub} Q_{J}$. Using the dual of [15, Proposition 5.6], we get that $g$ is a minimal left add $(T)$-approximation, $T \oplus Y$ is basic rigid, $Y$ is indecomposable and not isomorphic to $X$. Again by Proposition 4.1, we have that $Y \oplus T \oplus \tau(V) \oplus Q_{K}$ is a basic maximal rigid module in $\bmod \Lambda$. By [15] this is the mutation of $X \oplus T \oplus \tau(V) \oplus Q_{K}$ in the direction of $X$. This shows in particular that $f$ and $g$ are in fact add $\left(T \oplus \tau(V) \oplus Q_{K}\right)$-approximations, and that $Y$ is the unique indecomposable module in $\operatorname{Sub} Q_{J}$ non-isomorphic to $X$ and such that $T \oplus Y$ is complete rigid. By [15] we have another short exact sequence

$$
0 \rightarrow X \stackrel{i}{\rightarrow} T^{\prime \prime} \stackrel{h}{\rightarrow} Y \rightarrow 0
$$

where $h$ and $i$ are add $\left(T \oplus \tau(V) \oplus Q_{K}\right)$-approximations. Since Sub $Q_{J}$ is closed under extensions, $T^{\prime \prime}$ is in $\operatorname{Sub} Q_{J}$. Hence $h$ and $i$ are also add $(T)$-approximations, and in particular $T^{\prime \prime}$ does not depend on the choice of $V$. It also follows from [15, Corollary 6.5] that $\operatorname{dim} \operatorname{Ext}_{\Lambda}^{1}(X, Y)=1$, hence the short exact sequences (4), (5) are the unique non-split short exact sequences between $X$ and $Y$. To summarize, we have obtained the following

Proposition 8.1 Let $X \oplus T$ be a basic complete rigid module in Sub $Q_{J}$ with $X$ indecomposable and not Ext-projective. There exists a unique indecomposable module $Y \nsucceq X$ in $\operatorname{Sub} Q_{J}$ such that $Y \oplus T$ is a basic complete rigid module in $\operatorname{Sub} Q_{J}$. Moreover, $\operatorname{dimExt}_{\Lambda}^{1}(X, Y)=1$ and if (4), (5) are 
the unique non-split short exact sequences between $X$ and $Y$, then $f, g, h, i$ are minimal add $(T)$ approximations.

In the situation of the above Proposition, we say that $Y \oplus T$ is the mutation of $X \oplus T$ in the direction of $X$, and we write $\mu_{X}(X \oplus T)=Y \oplus T$. Let $U=X \oplus T$. Let $B=B\left(U \oplus \tau(V) \oplus Q_{K}\right)^{\circ}$ be the exchange matrix of the maximal rigid module $U \oplus \tau(V) \oplus Q_{K}$. The $\left(r-r_{K}\right) \times\left(r-r_{K}-n\right)$ submatrix of $B$ whose rows are labelled by the indecomposable summands of $U$ and columns by the indecomposable summands of $U \backslash L$ is called the exchange matrix of $U$, and denoted $B(U)^{\circ}$. By the discussion above, it contains all the information to calculate the mutations of $U$ in all the $r-r_{K}-n$ directions. Moreover, the submatrix of $B$ whose rows are labelled by the summands of $\tau(V) \oplus Q_{K}$ and columns by the indecomposable summands of $U \backslash L$ has all its entries equal to 0 . Taking into account the results of [15], this implies the

Proposition 8.2 Let $U$ be a basic complete rigid module in $\operatorname{Sub} Q_{J}$. Write $U=U_{1} \oplus \cdots \oplus U_{r-r_{K}}$, where the last $n$ summands are Ext-projective. Let $k \leqslant r-r_{K}-n$. We have

$$
B\left(\mu_{U_{k}}(U)\right)^{\circ}=\mu_{k}\left(B(U)^{\circ}\right),
$$

where in the right-hand side $\mu_{k}$ denotes the Fomin-Zelevinsky matrix mutation.

\section{Cluster algebra structure on $\mathbb{C}\left[N_{K}\right]$}

9.1 The next proposition relates the coordinate ring $\mathbb{C}\left[N_{K}\right]$ to the subcategory $\operatorname{Sub} Q_{J}$.

Proposition 9.1 The algebra $\mathbb{C}\left[N_{K}\right]$ is isomorphic to the subspace of $\mathbb{C}[N]$ spanned by the set

$$
\left\{\varphi_{M} \mid M \in \operatorname{Sub} Q_{J}\right\}
$$

Proof - By 2.5, $\mathbb{C}\left[N_{K}\right]$ is isomorphic to the (non direct) sum of the subspaces

$$
\operatorname{pr}_{I}(L(\lambda)), \quad\left(\lambda \in \Pi_{J}\right)
$$

of $\mathbb{C}[N]$. It was shown in [13, Th. 3] that, if $\lambda=\sum_{j \in J} a_{j} \varpi_{j}$ and $Q(\lambda)=\oplus_{j \in J} Q_{j}^{\oplus a_{j}}$, then $\operatorname{pr}_{I}(L(\lambda))$ is spanned by the $\varphi_{M}$ where $M$ runs over all submodules of $Q(\lambda)$. The result follows.

9.2 Following 7.2 , we introduce the set $R\left(w_{0}, K\right)$ of reduced words $\mathbf{i}=\left(\mathbf{i}^{\prime}, \mathbf{i}^{\prime \prime}\right)$ for $w_{0}$ starting with a reduced word $\mathbf{i}^{\prime}$ of $w_{0}^{K}$. By Proposition 7.3 , if $\mathbf{i} \in R\left(w_{0}, K\right)$, the basic complete rigid module $T_{\mathbf{i}}$ has a unique direct summand which is a complete rigid module in $\operatorname{Sub} Q_{J}$. We shall denote this summand by $U_{\mathbf{i}}$. If $\mathbf{j}=\left(\mathbf{j}^{\prime}, \mathbf{j}^{\prime \prime}\right)$ is another word in $R\left(w_{0}, K\right)$, we can pass from $\mathbf{i}^{\prime}$ to $\mathbf{j}^{\prime}$ by a sequence of 2-moves and 3-moves, and similarly from $\mathbf{i}^{\prime \prime}$ to $\mathbf{j}^{\prime \prime}$ by a sequence of 2-moves and 3-moves. Using [4, Remark 2.14], this implies that $T_{\mathbf{i}}$ and $T_{\left(\mathbf{j}^{\prime}, \mathbf{i}^{\prime \prime}\right)}$ are connected by a sequence of mutations. Moreover, the definition of the modules $T_{\mathbf{i}}$ shows that all these mutations will leave unchanged the direct summand $U_{\mathbf{i}} \in \operatorname{Sub} Q_{J}$. Similarly, $T_{\left(\mathbf{j}^{\prime}, \mathbf{i}^{\prime \prime}\right)}$ and $T_{\mathbf{j}}$ are connected by a sequence of mutations and it is plain that they all take place in $\operatorname{Sub} Q_{J}$. Hence the modules $U_{\mathbf{i}}$ and $U_{\mathbf{j}}$ are connected by a sequence of mutations in $\operatorname{Sub} Q_{J}$. To summarize, we have proved that 
Lemma 9.2 The set $\mathscr{R}_{J}$ of basic complete rigid modules in $\operatorname{Sub} Q_{J}$ which can be reached from $U_{\mathbf{i}}$ by a finite sequence of mutations does not depend on the choice of $\mathbf{i} \in R\left(w_{0}, K\right)$.

Now, exactly as in [15], we can project $\mathscr{R}_{J}$ on $\mathbb{C}\left[N_{K}\right]$ using the map $M \mapsto \varphi_{M}$. More precisely, put $d_{K}=r-r_{K}=\operatorname{dim} N_{K}$, and for $T=T_{1} \oplus \cdots \oplus T_{d_{K}} \in \mathscr{R}_{J}$, let $\mathbf{x}(T)=\left(\varphi_{T_{1}}, \ldots, \varphi_{T_{d_{K}}}\right)$. The next Theorem follows from the results of Section 8 and from the multiplication formula of [16].

Theorem 9.3 (i) $\left\{\mathbf{x}(T) \mid T \in \mathscr{R}_{J}\right\}$ is the set of clusters of a cluster algebra $\mathscr{A}_{J} \subseteq \mathbb{C}\left[N_{K}\right]$ of rank $d_{K}-n$.

(ii) The coefficient ring of $\mathscr{A}_{J}$ is the ring of polynomials in the $n$ variables $\varphi_{L_{i}}(i \in I)$.

(iii) All the cluster monomials belong to the dual semicanonical basis of $\mathbb{C}\left[N_{K}\right]$, and are thus linearly independent.

9.3 We now proceed to describe in detail initial seeds for $\mathscr{A}_{J}$.

9.3.1 Let $\mathbf{i}=\left(i_{1}, \ldots, i_{r}\right) \in R\left(w_{0}, K\right)$. Put $i_{-m}=m$ for $m=1, \ldots, n$. As in 6.3, set $u_{\leqslant m}=s_{i_{1}} \cdots s_{i_{m}}$ if $m=1, \ldots, r$, and $u_{\leqslant m}=e$ the unit element of $W$ if $m<0$. Then, by Corollary 6.2, the cluster of $\mathbb{C}[N]$ obtained by projecting $T_{\mathbf{i}}^{\dagger}$ consists of the $\mathrm{r}$ functions

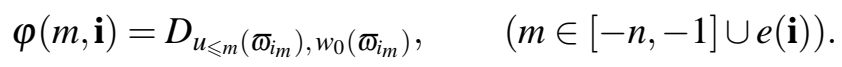

Moreover, $T_{\mathbf{i}}^{\dagger}=S T_{\mathbf{i}}$, where, as seen in 6.3, $T_{\mathbf{i}}$ projects on one of the initial seeds of [4]. By [15] the exchange matrix of a cluster seed of $\mathbb{C}[N]$ is a submatrix of the matrix of the Ringel form of the endomorphism algebra of the corresponding complete rigid module. It follows from 5.4 that the exchange matrix of the seeds corresponding to $T_{\mathbf{i}}$ and $T_{\mathbf{i}}^{\dagger}$ are given by the same combinatorial rule, which is described in [4]. We shall recall it for the convenience of the reader.

9.3.2 For $m \in[-n,-1] \cup e(\mathbf{i})$, let $m^{+}=\min \left\{l \in[1, r] \mid l>m\right.$ and $\left.i_{l}=i_{m}\right\}$. Next, one defines a quiver $\Gamma_{\mathbf{i}}$ with set of vertices $[-n,-1] \cup e(\mathbf{i})$. Assume that $m$ and $l$ are vertices such that $m<l$ and $\{m, l\} \cap e(\mathbf{i}) \neq \emptyset$. There is an arrow $m \rightarrow l$ in $\Gamma_{\mathbf{i}}$ if and only if $m^{+}=l$, and there is an arrow $l \rightarrow m$ if and only if $l<m^{+}<l^{+}$and $a_{i_{m}, i_{l}}<0$. Here, $\left(a_{i j}\right)_{1 \leqslant i, j \leqslant n}$ denotes the Cartan matrix of the root system of $G$. By definition these are all the arrows of $\Gamma_{\mathbf{i}}$. Now define an $r \times(r-n)$-matrix $B(\mathbf{i})=\left(b_{m l}\right)$ as follows. The columns of $B(\mathbf{i})$ are indexed by $e(\mathbf{i})$, and the rows by $e(\mathbf{i}) \cup[-n,-1]$. Set

$$
b_{m l}= \begin{cases}1 & \text { if there is an arrow } m \rightarrow l \text { in } \Gamma_{\mathbf{i}}, \\ -1 & \text { if there is an arrow } l \rightarrow m \text { in } \Gamma_{\mathbf{i}}, \\ 0 & \text { otherwise. }\end{cases}
$$

9.3.3 Finally, as in 7.2 for $k \in K$ let $t_{k}=\max \left\{t \leqslant r_{K} \mid i_{t}=k\right\}$. If $j \in J$, set $t_{j}=-j$. It follows from 7.2 that

$$
\left.\left.\mathbf{x}\left(U_{\mathbf{i}}\right)=\{\varphi(m, \mathbf{i}) \mid m \in] r_{K}, r\right] \cap e(\mathbf{i})\right\} \cup\left\{\varphi\left(t_{i}, \mathbf{i}\right) \mid i \in I\right\} .
$$

This is the cluster of our initial seed for $\mathscr{A}_{J}$. Here the first subset consists of the $r-r_{K}-n$ cluster variables. The second subset consisting of the $n$ generators of the coefficient ring is contained in 


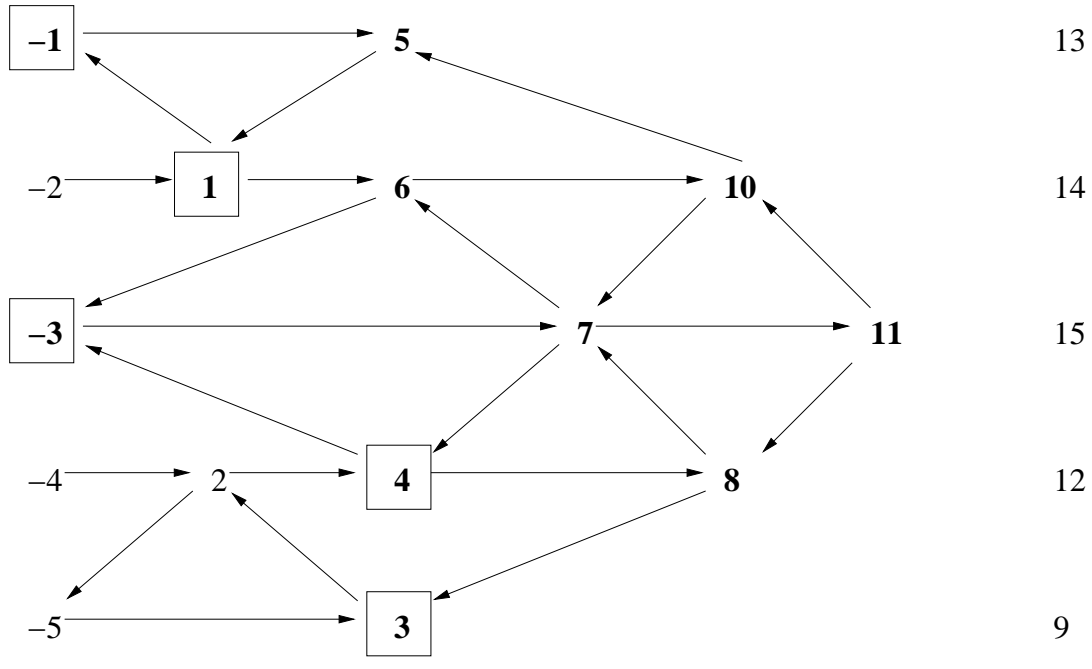

Figure 1: The graph $\Gamma_{\mathbf{i}}$.

every cluster. The corresponding exchange matrix is the submatrix of $B(\mathbf{i})$ with columns labelled by $\left.] r_{K}, r\right] \cap e(\mathbf{i})$ and rows labelled by (]$\left.\left.r_{K}, r\right] \cap e(\mathbf{i})\right) \cup\left\{t_{i} \mid i \in I\right\}$. Let us denote it by $B(\mathbf{i}, J)$. Then our construction yields

Proposition 9.4 For every $\mathbf{i} \in R\left(w_{0}, K\right)$, the pair $\left(\mathbf{x}\left(U_{\mathbf{i}}\right), B(\mathbf{i}, J)\right)$ is an initial seed of the cluster algebra $\mathscr{A}_{J} \subseteq \mathbb{C}\left[N_{K}\right]$.

Example 9.5 Let $G$ be of type $A_{5}$, that is, $G=S L_{6}$. Then $r=15$. Take $J=\{1,3\}$. Then we have $K=\{2,4,5\}$ and $r_{K}=4$. The word $\mathbf{i}=(\mathbf{2}, \mathbf{4}, \mathbf{5}, \mathbf{4}, 1,2,3,4,5,2,3,4,1,2,3)$ belongs to $R\left(w_{0}, K\right)$ : the subword $(2,4,5,4)$ is a reduced word for $w_{0}^{K}$. We have

$$
e(\mathbf{i})=\{1,2,3,4,5,6,7,8,10,11\} .
$$

The graph $\Gamma_{\mathbf{i}}$ is shown in Figure 11 The vertices 5,6,7,8,10,11 (in bold type) represent the cluster variables of the initial seed of $\mathscr{A}_{J}$, and the vertices $-1,1,-3,4,3$ (in squares) represent the generators of the coefficient ring. The exchange matrix is

$$
B(\mathbf{i}, J)=\left(\begin{array}{cccccc}
0 & 0 & 0 & 0 & -1 & 0 \\
0 & 0 & -1 & 0 & 1 & 0 \\
0 & 1 & 0 & -1 & -1 & 1 \\
0 & 0 & 1 & 0 & 0 & -1 \\
1 & -1 & 1 & 0 & 0 & -1 \\
0 & 0 & -1 & 1 & 1 & 0 \\
\hline 1 & 0 & 0 & 0 & 0 & 0 \\
-1 & 1 & 0 & 0 & 0 & 0 \\
0 & -1 & 1 & 0 & 0 & 0 \\
0 & 0 & -1 & 1 & 0 & 0 \\
0 & 0 & 0 & -1 & 0 & 0
\end{array}\right),
$$

where the rows of $B(\mathbf{i}, J)$ are labelled by $\{5,6,7,8,10,11,-1,1,-3,4,3\}$ and the columns by $\{5,6,7,8,10,11\}$. We have

$$
\varphi(5, \mathbf{i})=D_{3,6}, \quad \varphi(6, \mathbf{i})=D_{23,56}, \quad \varphi(7, \mathbf{i})=D_{236,456}, \quad \varphi(8, \mathbf{i})=D_{2356,3456},
$$




$$
\begin{gathered}
\varphi(10, \mathbf{i})=D_{36,56}, \quad \varphi(11, \mathbf{i})=D_{356,456}, \quad \varphi(-1, \mathbf{i})=D_{1,6}, \quad \varphi(1, \mathbf{i})=D_{13,56} \\
\varphi(-3, \mathbf{i})=D_{123,456}, \quad \varphi(4, \mathbf{i})=D_{1236,3456}, \quad \varphi(3, \mathbf{i})=D_{12356,23456}
\end{gathered}
$$

Here, since we are in type $A_{5}$, the generalized minors are simply minors of the upper unitriangular $6 \times 6$ matrix of coordinate functions on $N$. More precisely, for $w$ in the symmetric group $\mathfrak{S}_{6}$ the weight $w\left(\varpi_{i}\right)$ indexing a generalized minor can be identified with the subset $w([1, i])$ of $[1,6]$ of row or column indices of the minor. This is the convention we have used.

9.4 We end this section by stating the following

Conjecture 9.6 We have $\mathscr{A}_{J}=\mathbb{C}\left[N_{K}\right]$.

The conjecture will be proved for $G$ of type $A_{n}$ and of type $D_{4}$ in 10.4 It will also be proved for $J=\{n\}$ in type $D_{n}$ in Section 12 , and for $J=\{1\}$ in type $D_{5}$ in Section 13 .

\section{Cluster algebra structure on $\mathbb{C}\left[B_{K}^{-} \backslash G\right]$}

In this section we lift the cluster algebra $\mathscr{A}_{J} \subseteq \mathbb{C}\left[N_{K}\right]$ to a cluster algebra $\widetilde{\mathscr{A}_{J}} \subseteq \mathbb{C}\left[B_{K}^{-} \backslash G\right]$.

10.1 Let $(\mathbf{x}(T), B)$ denote a seed of $\mathscr{A}_{J}$. Let $x_{k}=\varphi_{T_{k}}$ be a cluster variable in $\mathbf{x}(T)$, and denote by $x_{k}^{*}$ the cluster variable obtained by mutation in direction $k$. The exchange relation between $x_{k}$ and $x_{k}^{*}$ is of the form

$$
x_{k} x_{k}^{*}=M_{k}+N_{k},
$$

where $M_{k}$ and $N_{k}$ are monomials in the variables of $\mathbf{x}(T)-\left\{x_{k}\right\}$. We have two exact sequences in $\operatorname{Sub} Q_{J}$

$$
0 \rightarrow T_{k} \rightarrow X_{k} \rightarrow T_{k}^{*} \rightarrow 0, \quad 0 \rightarrow T_{k}^{*} \rightarrow Y_{k} \rightarrow T_{k} \rightarrow 0,
$$

where $X_{k}, Y_{k} \in \operatorname{add} T$, and

$$
x_{k}^{*}=\varphi_{T_{k}^{*}}, \quad M_{k}=\varphi_{X_{k}}, \quad N_{k}=\varphi_{Y_{k}} .
$$

The following proposition is a particular case of a result in [18].

Proposition 10.1 With the above notation, for every $j \in J$ we have

$$
\operatorname{dim}_{\operatorname{Hom}}\left(S_{j}, T_{k}\right)+\operatorname{dim}_{H_{0}}\left(S_{j}, T_{k}^{*}\right)=\max \left\{\operatorname{dim}_{\operatorname{Hom}}\left(S_{j}, X_{k}\right), \operatorname{dim} \operatorname{Hom}_{\Lambda}\left(S_{j}, Y_{k}\right)\right\} .
$$

Recall from Section 2.5 the definition of $a_{j}(f)$ for $f \in \mathbb{C}\left[N_{K}\right]$. If $f=\varphi_{M}$ for some $\Lambda$-module $M$, it follows from [13] that $a_{j}(f)=\operatorname{dimHom}_{\Lambda}\left(S_{j}, M\right)$, the multiplicity of $S_{j}$ in the socle of $M$. Putting together Proposition 10.1 and Lemma 2.5 we thus have

$$
\widetilde{x_{k}} \widetilde{x_{k}^{*}}=\mu_{k} \widetilde{M_{k}}+v_{k} \widetilde{N_{k}},
$$

where $\mu_{k}$ and $v_{k}$ are monomials in the variables $\Delta_{\varpi_{j}, \varpi_{j}}(j \in J)$ without common divisor. More precisely,

$$
\mu_{k}=\prod_{j \in J} \Delta_{\varpi_{j}, \Phi_{j}}^{\alpha_{j}}, \quad v_{k}=\prod_{j \in J} \Delta_{\varpi_{j}, \bar{\varpi}_{j}}^{\beta_{j}},
$$

where

$$
\begin{aligned}
& \alpha_{j}=\max \left\{0, \operatorname{dim}_{H_{0}}\left(S_{j}, Y_{k}\right)-\operatorname{dim}_{\operatorname{Hom}}\left(S_{j}, X_{k}\right)\right\}, \\
& \beta_{j}=\max \left\{0, \operatorname{dim}_{\operatorname{Hom}_{\Lambda}}\left(S_{j}, X_{k}\right)-\operatorname{dim}_{\operatorname{Hom}_{\Lambda}}\left(S_{j}, Y_{k}\right)\right\} .
\end{aligned}
$$


10.2 It follows that the elements $\widetilde{x}$ where $x$ runs through the set of cluster variables of $\mathscr{A}_{J}$ form the cluster variables of a new cluster algebra contained in $\mathbb{C}\left[B_{K}^{-} \backslash G\right]$. More precisely, for $T=$ $T_{1} \oplus \cdots \oplus T_{d_{K}} \in \mathscr{R}_{J}$, let $\widetilde{\mathbf{x}(T)}=\left\{\widetilde{\varphi_{T_{1}}}, \ldots, \widetilde{\varphi_{T_{d_{K}}}}\right\} \sqcup\left\{\Delta_{\varpi_{j}, \Phi_{j}} \mid j \in J\right\}$.

Theorem 10.2 (i) $\left\{\widetilde{\mathbf{x}(T)} \mid T \in \mathscr{R}_{J}\right\}$ is the set of clusters of a cluster algebra $\widetilde{\mathscr{A}_{J}} \subseteq \mathbb{C}\left[B_{K}^{-} \backslash G\right]$ of rank $d_{K}-n$.

(ii) The coefficient ring of $\widetilde{\mathscr{A}_{J}}$ is the ring of polynomials in the $n+|J|$ variables $\widetilde{\varphi_{L_{i}}}(i \in I)$ and $\Delta_{\varpi_{j}, \varpi_{j}}(j \in J)$.

(iii) The exchange matrix $\widetilde{B}$ attached to $\widetilde{\mathbf{x}(T)}$ is obtained from the exchange matrix $B$ of $\mathbf{x}(T)$ by adding $|J|$ new rows (in the non-principal part) labelled by $j \in J$ encoding the monomials $\mu_{k}$ and $v_{k}$ in Equations (7) and (8). More precisely, the entry in column $k$ and row $j$ is equal to

$$
b_{j k}=\operatorname{dim} \operatorname{Hom}_{\Lambda}\left(S_{j}, X_{k}\right)-\operatorname{dim} \operatorname{Hom}_{\Lambda}\left(S_{j}, Y_{k}\right) .
$$

Proof - The only thing to be proved is that the enlarged exchange matrices $\widetilde{B}$ of (iii) follow the matrix mutation rule of Fomin and Zelevinsky. To do that, let us introduce some notation. Let $b_{i k}$ denote the entry of $\widetilde{B}=\widetilde{B}(T)$ on row $i$ and column $k$. Here $k$ runs through $\left\{1, \ldots, d_{K}-n\right\}$ and $i$ runs through $\left\{1, \ldots, d_{K}\right\} \cup J$. For $k \in\left\{1, \ldots, d_{K}-n\right\}$ the mutation of $T$ in direction $k$ comes from the two short exact sequences

$$
0 \rightarrow T_{k} \rightarrow X_{k} \rightarrow T_{k}^{*} \rightarrow 0, \quad 0 \rightarrow T_{k}^{*} \rightarrow Y_{k} \rightarrow T_{k} \rightarrow 0 .
$$

Fix $l \in\left\{1, \ldots, d_{K}-n\right\}$ and let us consider the mutation $\mu_{l}(T)=T_{l}^{*} \oplus T / T_{l}$. We denote by $\widetilde{B}^{\prime}=\left[b_{i k}^{\prime}\right]$ the exchange matrix attached to $\mu_{l}(T)$, as defined in (iii). We have to show that $b_{i k}^{\prime}=-b_{i k}$ if $i$ or $k$ is equal to $l$, and otherwise

$$
b_{i k}^{\prime}=b_{i k}+\frac{b_{i l}\left|b_{l k}\right|+\left|b_{i l}\right| b_{l k}}{2} .
$$

(a) By construction this holds for $i \in\left\{1, \ldots, d_{K}\right\}$, so we shall now assume that $i$ is an element $j$ of $J$.

If $k=l$, the two short exact sequences used to define $b_{j k}$ and $b_{j k}^{\prime}$ are the same but they are interchanged, so it is plain that $b_{j k}^{\prime}=-b_{j k}$.

From now on we assume that $k \neq l$. Let

$$
0 \rightarrow T_{k} \rightarrow X_{k}^{\prime} \rightarrow^{\prime} T_{k}^{*} \rightarrow 0, \quad 0 \rightarrow{ }^{\prime} T_{k}^{*} \rightarrow Y_{k}^{\prime} \rightarrow T_{k} \rightarrow 0,
$$

denote the two short exact sequences inducing the mutation of $\mu_{l}(T)$ in direction $k$. If $b_{l k}=0$ then $b_{i k}^{\prime}=b_{i k}$ for all $i \in\left\{1, \ldots, d_{K}\right\}$. It follows that $X_{k}^{\prime}=X_{k}$ and $Y_{k}^{\prime}=Y_{k}$, hence ${ }^{\prime} T_{k}^{*}=T_{k}^{*}, b_{j k}^{\prime}=b_{j k}$, and the result is proved. Hence we can assume that $b_{l k} \neq 0$.

Assume that $b_{l k}>0$. For $X, Y \in \bmod \Lambda$ we shall use the shorthand notation

$$
[X, Y]=\operatorname{dim} \operatorname{Hom}_{\Lambda}(X, Y) .
$$

We have

$$
\left[S_{j}, X_{k}\right]-\left[S_{j}, Y_{k}\right]=-\sum_{b_{i k}<0} b_{i k}\left[S_{j}, T_{i}\right]-\sum_{b_{i k}>0} b_{i k}\left[S_{j}, T_{i}\right]=-\sum_{i} b_{i k}\left[S_{j}, T_{i}\right],
$$

where the sums are over $i \in\left\{1, \ldots, d_{K}\right\}$. Now, for $i \in\left\{1, \ldots, d_{K}\right\}$, it follows from (a) that $b_{i k}^{\prime}=b_{i k}$ unless $b_{i l}>0$ in which case $b_{i k}^{\prime}=b_{i k}+b_{i l} b_{l k}$. 

that

(b) Let us further assume that $b_{j l}>0$. This implies that $\left[S_{j}, T_{l}^{*}\right]=\left[S_{j}, X_{l}\right]-\left[S_{j}, T_{l}\right]$. Now, using

$$
X_{k}^{\prime}=\left(T_{l}^{*}\right)^{b_{l k}} \oplus \bigoplus_{\substack{i \neq l \\ b_{i k}^{\prime}<0}} T_{i}^{\left(-b_{i k}^{\prime}\right)}, \quad Y_{k}^{\prime}=\bigoplus_{b_{i k}^{\prime}>0} T_{i}^{b_{i k}^{\prime}},
$$

we calculate

$$
\begin{gathered}
{\left[S_{j}, X_{k}^{\prime}\right]=b_{l k}\left(\left[S_{j}, X_{l}\right]-\left[S_{j}, T_{l}\right]\right)-\sum_{\substack{b_{i l}>0 \\
b_{i k}+b_{i l} b_{l k}<0}}\left(b_{i k}+b_{i l} b_{l k}\right)\left[S_{j}, T_{i}\right]-\sum_{\substack{b_{i l} \leqslant 0 \\
b_{i k}<0}} b_{i k}\left[S_{j}, T_{i}\right],} \\
{\left[S_{j}, Y_{k}^{\prime}\right]=\sum_{\substack{b_{i l}>0 \\
b_{i k}+b_{i l} b_{l k}>0}}\left(b_{i k}+b_{i l} b_{l k}\right)\left[S_{j}, T_{i}\right]+\sum_{\substack{b_{i l} \leqslant 0 \\
b_{i k}>0 \\
i \neq l}} b_{i k}\left[S_{j}, T_{i}\right] .}
\end{gathered}
$$

This implies that

$$
\begin{aligned}
{\left[S_{j}, X_{k}^{\prime}\right]-\left[S_{j}, Y_{k}^{\prime}\right] } & =b_{l k}\left(\left[S_{j}, X_{l}\right]-\left[S_{j}, T_{l}\right]\right)-\sum_{b_{i l}>0}\left(b_{i k}+b_{i l} b_{l k}\right)\left[S_{j}, T_{i}\right]-\sum_{\substack{b_{i l} \leqslant 0 \\
i \neq l}} b_{i k}\left[S_{j}, T_{i}\right] \\
= & b_{l k}\left[S_{j}, X_{l}\right]-\sum_{b_{i l}>0}\left(b_{i k}+b_{i l} b_{l k}\right)\left[S_{j}, T_{i}\right]-\sum_{b_{i l} \leqslant 0} b_{i k}\left[S_{j}, T_{i}\right] \\
& =-\sum_{i} b_{i k}\left[S_{j}, T_{i}\right]+b_{l k}\left(\left[S_{j}, X_{l}\right]-\sum_{b_{i l}>0} b_{i l}\left[S_{j}, T_{i}\right]\right)
\end{aligned}
$$

Hence

$$
\left[S_{j}, X_{k}^{\prime}\right]-\left[S_{j}, Y_{k}^{\prime}\right]=\left[S_{j}, X_{k}\right]-\left[S_{j}, Y_{k}\right]+b_{l k}\left(\left[S_{j}, X_{l}\right]-\left[S_{j}, Y_{l}\right]\right),
$$

that is $b_{j k}^{\prime}=b_{j k}+b_{l k} b_{j l}$, as required.

(c) Assume now that $b_{j l} \leqslant 0$. This implies that $\left[S_{j}, T_{l}^{*}\right]=\left[S_{j}, Y_{l}\right]-\left[S_{j}, T_{l}\right]$, and the same calculation as above now gives

$$
\left[S_{j}, X_{k}^{\prime}\right]-\left[S_{j}, Y_{k}^{\prime}\right]=\left[S_{j}, X_{k}\right]-\left[S_{j}, Y_{k}\right]
$$

that is, $b_{j k}^{\prime}=b_{j k}$. This finishes the proof when $b_{l k}>0$. The case $b_{l k}<0$ is entirely similar.

Note that $d_{K}+|J|$ is equal to the dimension of the multi-cone over $B_{K}^{-} \backslash G$. Note also that the clusters of $\mathscr{A}_{J}$ and $\widetilde{\mathscr{A}_{J}}$ are in natural one-to-one correspondence, and the principal parts of the exchange matrices of two corresponding clusters are the same. This shows that $\mathscr{A}_{J}$ and $\widetilde{\mathscr{A}_{J}}$ are both of finite cluster type or of infinite cluster type, and if they are of finite type, their types are the same.

Example 10.3 We continue Example 9.5. Let us denote for short by $x_{m}$ the functions $\varphi(m, \mathbf{i})$ of Example 9.5. Thus we have 11 minors

$$
x_{5}, x_{6}, x_{7}, x_{8}, x_{10}, x_{11}, x_{-1}, x_{1}, x_{-3}, x_{4}, x_{3}
$$

in $\mathbb{C}\left[N_{K}\right]$. It is straightforward to calculate their lifts to $\mathbb{C}\left[B_{K}^{-} \backslash G\right]$. One gets

$$
\begin{aligned}
& \widetilde{x_{5}}=\Delta_{126}, \quad \widetilde{x_{6}}=\Delta_{156}, \quad \widetilde{x_{7}}=\Delta_{145}, \quad \widetilde{x_{8}}=\Delta_{134}, \quad \widetilde{x_{10}}=\Delta_{125}, \quad \widetilde{x_{11}}=\Delta_{124}, \\
& \widetilde{x_{-1}}=\Delta_{6}, \quad \widetilde{x_{1}}=\Delta_{2} \Delta_{156}-\Delta_{1} \Delta_{256}, \quad \widetilde{x_{-3}}=\Delta_{456}, \quad \widetilde{x_{4}}=\Delta_{345}, \quad \widetilde{x_{3}}=\Delta_{234} .
\end{aligned}
$$


In these formulas, all minors are flag minors, hence we have indicated only their column indices. For example, using the fact that $D_{I, J}$ is a minor of an upper unitriangular matrix, we have,

$$
x_{8}=D_{2356,3456}=D_{23,34}=D_{123,134} \text {. }
$$

Hence, $\widetilde{x_{8}}=\Delta_{123,134}=\Delta_{134}$. A more interesting example is

$$
x_{1}=D_{13,56}=D_{1,2} D_{23,56}-D_{123,256}=D_{1,2} D_{123,156}-D_{123,256} .
$$

Hence,

$$
\widetilde{x_{1}}=\Delta_{1,2} \Delta_{123,156}-\Delta_{1,1} \Delta_{123,256}=\Delta_{2} \Delta_{156}-\Delta_{1} \Delta_{256} .
$$

Note that $\widetilde{x_{1}}$ cannot be written as a flag minor on $G$. (In representation theoretical terms, this is because the socle of the indecomposable rigid $\Lambda$-module $L_{4}$ attached to $x_{1}$ is not simple : it is isomorphic to $S_{1} \oplus S_{3}$.) Since $\widetilde{x_{1}}$ is one of the generators of the coefficient ring of $\widetilde{\mathscr{A}_{J}}$ (that is, $L_{4}=P_{4} / S_{2}$ is projective in Sub $Q_{J}$, see Proposition 3.2), it belongs to every seed of the cluster structure of $\widetilde{\mathscr{A}_{J}}$. This example shows that, in contrast to the case of the full flag variety, the cluster algebra $\widetilde{\mathscr{A}}_{J}$ of a partial flag variety may have no seed consisting entirely of flag minors.

Finally, the exchange matrix for this seed of $\widetilde{\mathscr{A}_{J}}$ is

$$
\widetilde{B}(\mathbf{i}, J)=\left(\begin{array}{cccccc}
0 & 0 & 0 & 0 & -1 & 0 \\
0 & 0 & -1 & 0 & 1 & 0 \\
0 & 1 & 0 & -1 & -1 & 1 \\
0 & 0 & 1 & 0 & 0 & -1 \\
1 & -1 & 1 & 0 & 0 & -1 \\
0 & 0 & -1 & 1 & 1 & 0 \\
\hline 1 & 0 & 0 & 0 & 0 & 0 \\
-1 & 1 & 0 & 0 & 0 & 0 \\
0 & -1 & 1 & 0 & 0 & 0 \\
0 & 0 & -1 & 1 & 0 & 0 \\
0 & 0 & 0 & -1 & 0 & 0 \\
\hline 0 & -1 & 0 & 0 & 0 & 0 \\
0 & 0 & 0 & 0 & 0 & 1
\end{array}\right) .
$$

It is obtained from the matrix of Example 9.5 by adding the last two rows labelled by $\Delta_{1}$ and $\Delta_{123}$. The two nonzero entries in these rows correspond to the two exchange relations for $\widetilde{x_{6}}$ and $\widetilde{x_{11}}$. $\diamond$

10.3 We now show that in type $A_{n}$ our algebras $\widetilde{\mathscr{A}}_{\{j\}}$ coincide with the cluster algebras on coordinate rings of Grassmannians considered in [10] for $j=2$, and in [32] for general $j$. To do so, it is enough to check that one of our seed for $\mathscr{A}_{\{j\}}$ coincides with a seed of [32].

10.3.1 In the case of type $A_{n}$ and $J=\{j\}$, the rule described in Proposition 9.4 gives us a unique initial cluster for $\mathscr{A}_{\{j\}}$. Indeed, if $\mathbf{i}=\left(\mathbf{i}^{\prime}, \mathbf{i}^{\prime \prime}\right)$ and $\mathbf{j}=\left(\mathbf{j}^{\prime}, \mathbf{j}^{\prime \prime}\right)$ are two elements in $R\left(w_{0}, I-\{j\}\right)$, then $\mathbf{i}^{\prime \prime}$ and $\mathbf{j}^{\prime \prime}$ are related by a sequence of 2-moves, and the corresponding clusters are therefore equal. This unique initial cluster of $\mathscr{A}_{\{j\}}$ consists of the minors $D_{C}$ where $C$ belongs to the following list:

$$
\begin{gathered}
\{1, \ldots, j-1, j+1\},\{1, \ldots, j-1, j+2\}, \ldots,\{\mathbf{1}, \ldots, \mathbf{j}-\mathbf{1}, \mathbf{n}+\mathbf{1}\}, \\
\{1, \ldots, j-2, j, j+1\},\{1, \ldots, j-2, j+1, j+2\}, \ldots,\{\mathbf{1}, \ldots, \mathbf{j}-\mathbf{2}, \mathbf{n}, \mathbf{n}+\mathbf{1}\}, \\
\{1, \ldots, j-3, j-1, j, j+1\},\{1, \ldots, j-3, j, j+1, j+2\}, \ldots,\{\mathbf{1}, \ldots, \mathbf{j}-\mathbf{3}, \mathbf{n}-\mathbf{1}, \mathbf{n}, \mathbf{n}+\mathbf{1}\},
\end{gathered}
$$




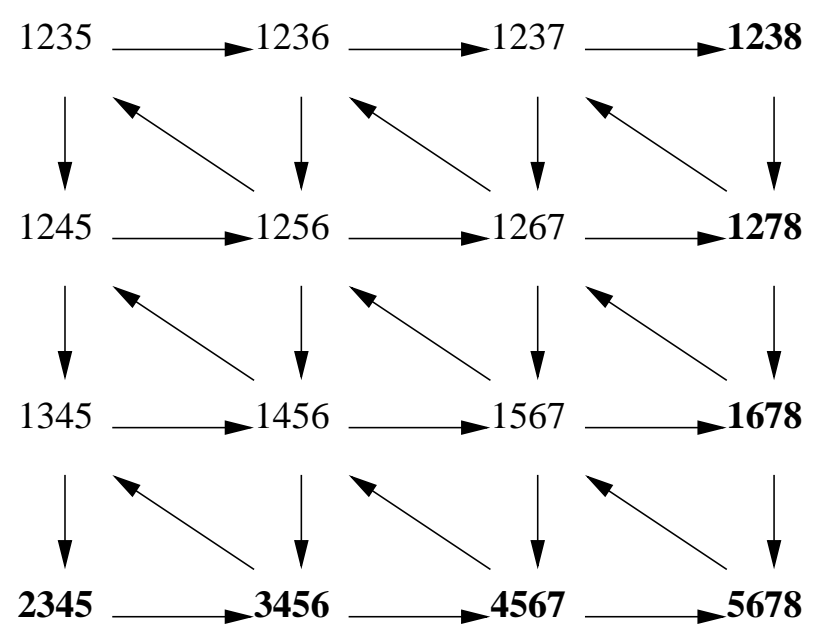

Figure 2: The grid for $n=7$ and $j=4$.

$$
\begin{gathered}
\ldots, \quad \cdots, \\
\{1,3, \ldots, j, j+1\},\{1,4, \ldots, j+1, j+2\}, \ldots,\{\mathbf{1}, \mathbf{n}-\mathbf{j}+\mathbf{3} \ldots, \mathbf{n}, \mathbf{n}+\mathbf{1}\} \\
\{\mathbf{2}, \ldots, \mathbf{j}+\mathbf{1}\},\{\mathbf{3}, \ldots, \mathbf{j}+\mathbf{2}\}, \ldots,\{\mathbf{n}-\mathbf{j}+\mathbf{2}, \ldots, \mathbf{n}+\mathbf{1}\} .
\end{gathered}
$$

The $n$ subsets in bold type correspond to generators of the coefficient ring, and therefore cannot be mutated. Here we use the same notation as in Example 10.3 for the flag minors in type $A$. Note that it is not obvious from Proposition 9.4 that all elements of this initial cluster can be written as flag minors $D_{C}$. It turns out to be true and not difficult to check in this special case. From the point of view of preprojective algebras, this case is special in the sense that all indecomposable summands of the rigid module $U_{\mathbf{i}}$ of 9.2 , which by construction have simple top, also have simple socle.

The graph describing the exchange matrix of this cluster has the shape of a rectangular grid with $j$ rows and $n-j+1$ columns. The vertices are the subsets $C$ displayed as above. There are horizontal right arrows, vertical down arrows and diagonal north-west arrows. For example, if $n=7$ and $j=4$, this graph is shown in Figure 2

We note that this initial seed coincides with the one described in [20, §3.3].

10.3.2 Let $\Delta_{C}$ denote the flag minor on $G$ corresponding to $D_{C}$. Thus, $\Delta_{C}=\widetilde{D_{C}}$. The initial cluster of $\widetilde{\mathscr{A}}_{\{j\}}$ lifting the cluster above consists of all $\Delta_{C}$ where $C$ runs over all sets in the above list, together with the new set $[1, j]=\{1, \ldots, j\}$. All the exchange relations are obtained by simply replacing each $D_{C}$ by the corresponding $\Delta_{C}$, except the exchange relation for $C=\{1, \ldots, j-1, j+$ 1) which reads

$$
\Delta_{\{1, \ldots, j-1, j+1\}} \Delta_{\{1, \ldots, j-2, j, j+2\}}=\Delta_{\{1, \ldots, j-1, j+2\}} \Delta_{\{1, \ldots, j-2, j, j+1\}}+\Delta_{[1, j]} \Delta_{\{1, \ldots, j-2, j+1, j+2\}} .
$$

One then checks that this coincides with one of the seeds of [32]. To do so, one has to construct a Postnikov arrangement [30], [32, §3] whose labelling is given by the list of subsets $C$ of our seed for $\widetilde{\mathscr{A}_{\{j\}}}$. This arrangement has a regular structure similar to a honeycomb. The central labelled cells are hexagonal and the border ones are quadrilateral. If the sets $C$ and $C^{\prime}$ are connected by an arrow in the grid of 10.3.1, then the cells labelled by $C$ and $C^{\prime}$ have a common vertex. For example, when $n=7$ and $j \equiv 4$, the "honeycomb" Postnikov arrangement is shown in Figure 3 . This finishes the proof that $\widetilde{\mathscr{A}}_{\{j\}}$ coincides with the cluster algebra of [32]. 


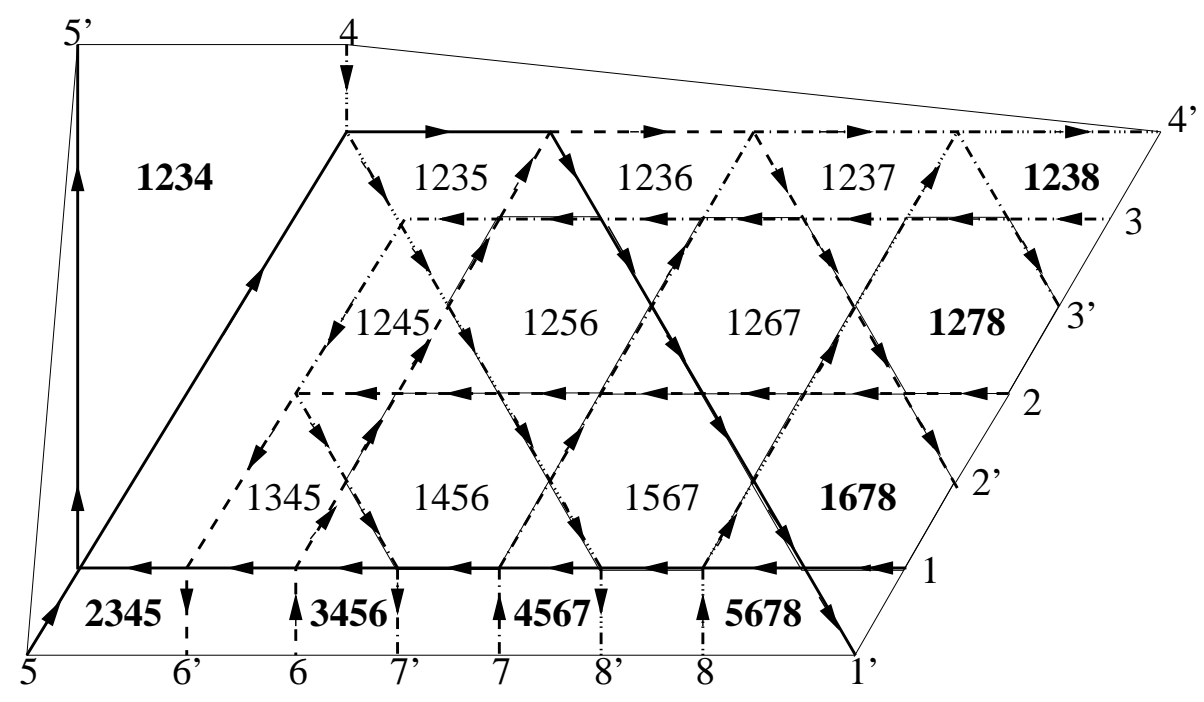

Figure 3: The honeycomb arrangement for $n=7$ and $j=4$.
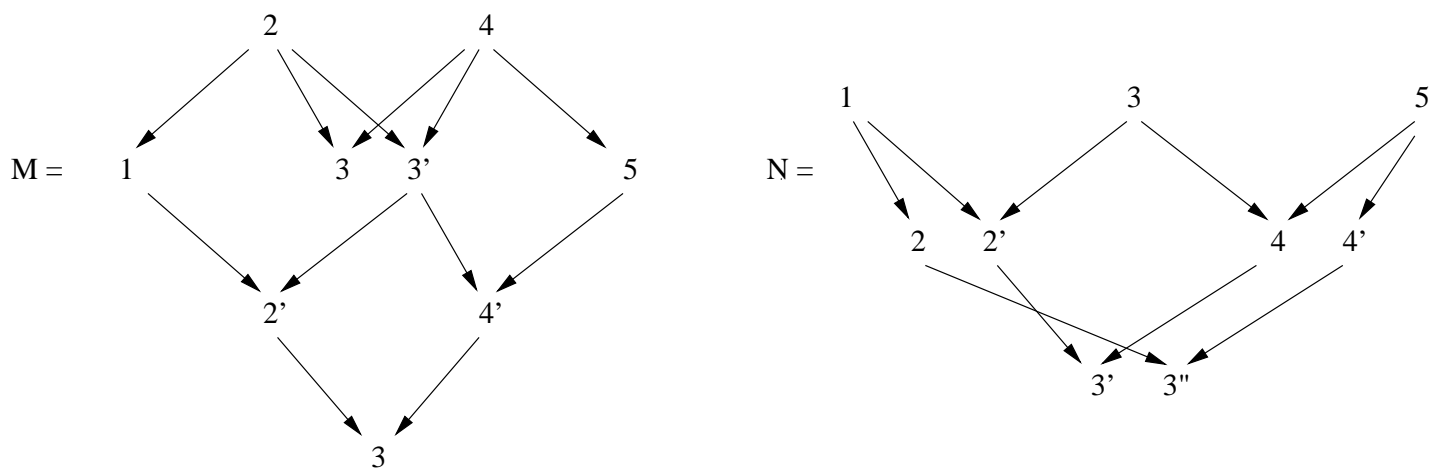

Figure 4: The rigid $\Lambda$-modules $M$ and $N$ in $\operatorname{Sub} Q_{3}$ for type $A_{5}$.

10.3.3 We think that our construction sheds a new light on the cluster algebra structures of the coordinate rings of Grassmannians. For example, in [32, Th. 6], Scott describes the two special cluster variables $X$ and $Y$ of $\mathbb{C}[\operatorname{Gr}(3,6)]$ which are not flag minors. In our setting

$$
X=\widetilde{\varphi_{M}}, \quad Y=\widetilde{\varphi_{N}},
$$

where $M$ and $N$ are the only two indecomposable rigid modules of $\operatorname{Sub} Q_{3}$ in type $A_{5}$ with a 2-dimensional socle $S_{3} \oplus S_{3}$. These modules are represented in Figure 4

10.4 Let $\Sigma_{J}$ be the multiplicative submonoid of $\widetilde{\mathscr{A}_{J}}$ generated by the set

$$
\left\{\Delta_{\varpi_{j}, \varpi_{j}} \mid j \in J \text { and } \varpi_{j} \text { is not minuscule }\right\} \text {. }
$$

Conjecture 10.4 The localizations of $\widetilde{\mathscr{A}_{J}}$ and $\mathbb{C}\left[B_{K}^{-} \backslash G\right]$ with respect to $\Sigma_{J}$ are equal.

Note that if $J$ is such that all the weights $\varpi_{j}(j \in J)$ are minuscule, then $\Sigma_{J}$ is trivial and the conjecture states that the algebras $\widetilde{\mathscr{A}_{J}}$ and $\mathbb{C}\left[B_{K}^{-} \backslash G\right]$ coincide without localization. This is in particular 
the case for every $J$ in type $A_{n}$. Note also that Conjecture 10.4 implies Conjecture 9.6 Indeed, by construction $\operatorname{pr}_{J}\left(\widetilde{\mathscr{A}_{J}}\right)=\mathscr{A}_{J}, \operatorname{pr}_{J}\left(\Sigma_{J}\right)=\{1\}$ and by $2.5, \operatorname{pr}_{J}\left(\mathbb{C}\left[B_{K}^{-} \backslash G\right]\right)=\mathbb{C}\left[N_{K}\right]$. We shall now prove Conjecture 10.4 in type $A_{n}$ and $D_{4}$.

10.4.1 We first remark that if the conjecture is true for every $\widetilde{\mathscr{A}}_{\{j\}}(j \in J)$, then it is true for $\widetilde{\mathscr{A}_{J}}$. Indeed, if $J^{\prime} \subset J$ and $K^{\prime}=I \backslash J$, there exist reduced words for $w_{0}$ of the form $\mathbf{i}=\left(\mathbf{i}^{\prime}, \mathbf{i}^{\prime \prime}, \mathbf{i}^{\prime \prime \prime}\right)$ with $\mathbf{i}^{\prime}$ a reduced word for $w_{0}^{K}$ and $\left(\mathbf{i}^{\prime}, \mathbf{i}^{\prime \prime}\right)$ a reduced word for $w_{0}^{K^{\prime}}$. This shows that the initial seed for $\widetilde{\mathscr{A}_{J}}$ associated with $\mathbf{i}$ will contain the initial seed for $\widetilde{\mathscr{A}_{J^{\prime}}}$ associated with $\mathbf{i}$. Hence $\widetilde{\mathscr{A}_{J^{\prime}}}$ is a subalgebra of $\widetilde{\mathscr{A}}_{J}$. In particular, $\widetilde{\mathscr{A}}_{\{j\}}$ is a subalgebra of $\widetilde{\mathscr{A}}_{J}$ for every $j \in J$. Suppose we know that $\mathbb{C}\left[B_{I \backslash\{j\}}^{-} \backslash G\right]$ is contained in the localization of $\widetilde{\mathscr{A}_{\{j\}}}$ with respect to $\Sigma_{\{j\}}$ for every $j \in J$. Then, since $\mathbb{C}\left[B_{K}^{-} \backslash G\right]=\bigoplus_{\lambda \in \Pi_{J}} L(\lambda)$ is generated (as a ring) by the subspaces $L\left(\varpi_{j}\right) \subset \mathbb{C}\left[B_{I \backslash\{j\}}^{-} \backslash G\right]$ we have that $\mathbb{C}\left[B_{K}^{-} \backslash G\right]$ is generated by the localized rings $\widetilde{\mathscr{A}_{\{j\}}}\left[\Sigma_{\{j\}}^{-1}\right]$, hence also by $\widetilde{\mathscr{A}_{J}}\left[\Sigma_{J}^{-1}\right]$. Therefore Conjecture 10.4 is satisfied.

10.4.2 In type $A_{n}$, the algebra $\mathbb{C}\left[B_{I \backslash\{j\}}^{-} \backslash G\right]$ is generated by the set of flag minors

$$
\Delta_{\varpi_{j}, w\left(\varpi_{j}\right)} \quad(w \in W) .
$$

Hence to prove the conjecture in this case it is enough to show that each of these minors belongs to $\widetilde{\mathscr{A}_{\{j\}}}$. In [32], Scott proves that all flag minors are cluster variables of $\widetilde{\mathscr{A}}_{\{j\}}$. It follows that Conjecture 10.4 is true in type $A$.

10.4.3 We now prove Conjecture 10.4 in type $D_{4}$. We choose to label by 3 the central node of the Dynkin diagram. By 10.4.1 it is enough to check the conjecture in the cases $J=\{1\},\{2\},\{3\}$ and $\{4\}$. Because of the order 3 diagram automorphism of $D_{4}$, the cases $J=\{1\},\{2\}$ and $\{4\}$ are identical. They are dealt with in detail in Section 12 (which studies more generally the case $J=\{n\}$ in type $D_{n}$ ). So we are left with $J=\{3\}$.

To prove the conjecture in this case we have to show that $\widetilde{\mathscr{A}_{\{3\}}}$ contains, up to localization by $\Delta_{\varpi_{3}, \varpi_{3}}$, a basis of the $G$-module $L\left(\varpi_{3}\right)$. This module has dimension 28 . The 24 generalized minors $\Delta_{\Phi_{3}, u\left(\Phi_{3}\right)}(u \in W)$ are extremal vectors of $L\left(\varpi_{3}\right)$, but we need 4 more vectors to get a basis. We shall use the dual semicanonical basis of $L\left(\varpi_{3}\right)$, obtained by lifting the dual semicanonical basis of the subspace $\operatorname{pr}_{\{3\}}\left(L\left(\varpi_{3}\right)\right)$ of $\mathbb{C}[N]$ via the map $x \mapsto \widetilde{x}$. This basis consists of elements of the form $\widetilde{\varphi_{M}}$, where $M$ runs through "generic" submodules of $Q_{3}$. It contains the 24 minors above, attached to 24 rigid submodules $M$. In particular,

$$
\Delta_{\bar{\omega}_{3}, \varpi_{3}}=\widetilde{\varphi_{0}}, \quad \Delta_{\bar{\omega}_{3}, w_{0}\left(\varpi_{3}\right)}=\widetilde{\varphi_{Q_{3}}},
$$

where $\mathbf{0}$ denotes the trivial submodule of $Q_{3}$. The 4 remaining vectors are $\widetilde{\varphi_{L_{1}}}, \widetilde{\varphi_{L_{2}}}, \widetilde{\varphi_{L_{4}}}$ and $\widetilde{\varphi_{M}(\lambda)}$, where

$$
M(\lambda), \quad(\lambda \in \mathbb{C}-\{0,1\}),
$$

denotes the 1-parameter family of $\Lambda$-modules represented in Figure 5 Note that, by construction, $\widetilde{\varphi_{L_{1}}}, \widetilde{\varphi_{L_{2}}}, \widetilde{\varphi_{L_{4}}}, \Delta_{\varpi_{3}, \Phi_{3}}$ and $\Delta_{\varpi_{3}, w_{0}\left(\varpi_{3}\right)}=\widetilde{\varphi_{Q_{3}}}$ belong to $\widetilde{\mathscr{A}_{\{3\}}}$, since they are the generators of the coefficient ring.

We choose $\mathbf{i}=(1,2,4,3,1,2,4,3,1,2,4,3) \in R\left(w_{0}, K\right)$ and consider the corresponding initial seed of $\widetilde{\mathscr{A}_{\{3\}}}$. The 5 cluster variables of this seed are

$$
z_{1}=\Delta_{\varpi_{3}, s_{3}\left(\varpi_{3}\right)}, \quad z_{2}=\Delta_{\bar{\varpi}_{3}, s_{1} s_{3}\left(\varpi_{3}\right)}, \quad z_{3}=\Delta_{\bar{\omega}_{3}, s_{2} s_{3}\left(\varpi_{3}\right)}, \quad z_{4}=\Delta_{\varpi_{3}, s_{4} s_{3}\left(\varpi_{3}\right)},
$$




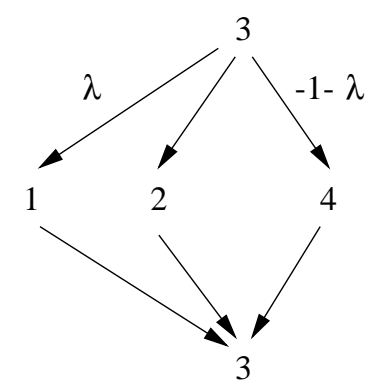

Figure 5: The one-parameter family $M(\lambda)$ in $\operatorname{Sub} Q_{3}$ for type $D_{4}$.

\begin{tabular}{|c|c|}
\hline$u$ & mutation sequence for $\Delta_{\bar{\omega}_{3}, u\left(\varpi_{3}\right)}$ \\
\hline$s_{2} s_{1} s_{3}$ & $\mu_{4} \mu_{1}$ \\
$s_{4} s_{1} s_{3}$ & $\mu_{3} \mu_{1}$ \\
$s_{4} s_{1} s_{3}$ & $\mu_{2} \mu_{1}$ \\
$s_{3} s_{2} s_{1} s_{3}$ & $\mu_{4}$ \\
$s_{3} s_{4} s_{1} s_{3}$ & $\mu_{3}$ \\
$s_{3} s_{4} s_{2} s_{3}$ & $\mu_{2}$ \\
$s_{1} s_{4} s_{2} s_{3}$ & $\mu_{4} \mu_{5} \mu_{1} \mu_{3} \mu_{2} \mu_{1}$ \\
$s_{4} s_{3} s_{2} s_{1} s_{3}$ & $\mu_{5} \mu_{1} \mu_{3} \mu_{2} \mu_{1}$ \\
$s_{2} s_{3} s_{4} s_{1} s_{3}$ & $\mu_{5} \mu_{1} \mu_{2} \mu_{4} \mu_{1}$ \\
$s_{1} s_{3} s_{4} s_{2} s_{3}$ & $\mu_{5} \mu_{1} \mu_{4} \mu_{3} \mu_{1}$ \\
$s_{3} s_{1} s_{4} s_{2} s_{3}$ & $\mu_{5} \mu_{4} \mu_{3} \mu_{2}$ \\
$s_{4} s_{3} s_{1} s_{4} s_{2} s_{3}$ & $\mu_{4} \mu_{5} \mu_{4} \mu_{3} \mu_{2}$ \\
$s_{2} s_{3} s_{1} s_{4} s_{2} s_{3}$ & $\mu_{3} \mu_{5} \mu_{4} \mu_{3} \mu_{2}$ \\
$s_{1} s_{3} s_{1} s_{4} s_{2} s_{3}$ & $\mu_{2} \mu_{5} \mu_{4} \mu_{3} \mu_{2}$ \\
$s_{2} s_{4} s_{3} s_{1} s_{4} s_{2} s_{3}$ & $\mu_{2} \mu_{5}$ \\
$s_{1} s_{2} s_{3} s_{1} s_{4} s_{2} s_{3}$ & $\mu_{3} \mu_{5}$ \\
$s_{1} s_{4} s_{3} s_{1} s_{4} s_{2} s_{3}$ & $\mu_{4} \mu_{5}$ \\
$s_{1} s_{2} s_{4} s_{3} s_{1} s_{4} s_{2} s_{3}$ & $\mu_{1} \mu_{5}$ \\
\hline
\end{tabular}

Table 1: Generalized minors $\Delta_{\varpi_{3}, u\left(\varpi_{3}\right)}$ obtained by cluster mutation.

and a variable $z_{5}$ of degree $2 \varpi_{3}$. One can then obtain by mutation the 18 other minors of the form $\Delta_{\bar{\varpi}_{3}, u\left(\varpi_{3}\right)}$, as shown in Table 1. Here, for example, the first row means that $\Delta_{\bar{\omega}_{3}, s_{2} s_{1} s_{3}\left(\varpi_{3}\right)}$ is the new cluster variable obtained by applying to the initial cluster a mutation $\mu_{1}$ with respect to the first variable, followed by a mutation $\mu_{4}$ with respect to the fourth variable.

It remains to show that $\widetilde{\varphi_{M(\lambda)}}$ also belongs to $\widetilde{\mathscr{A}_{\{3\}}}$, up to division by $\Delta_{\varpi_{3}, \Phi_{3}}$. For this, we use the multiplication formula for the functions $\varphi_{M}$ of [16]. Let $N_{1}$ and $N_{2}$ denote the $\Lambda$-modules

$$
N_{1}=1 \searrow_{3}^{2} \swarrow^{4,} \quad N_{2}=1 \searrow_{3} \swarrow^{2} \searrow_{3} \swarrow^{4 .}
$$

It is easy to check that $\widetilde{\varphi_{N_{1}}}=\Delta_{\widetilde{\omega}_{3}, s_{1} s_{4} s_{2} s_{3}\left(\varpi_{3}\right)}$ and that $\widetilde{\varphi_{N_{2}}}$ is the new cluster variable obtained from the initial cluster of $\widetilde{\mathscr{A}_{\{3\}}}$ via one mutation with respect to $z_{1}$. By [16] we have

$$
\varphi_{M(\lambda)}=\varphi_{S_{3}} \varphi_{N_{1}}-\varphi_{N_{2}}-\varphi_{L_{1}}-\varphi_{L_{2}}-\varphi_{L_{4}} .
$$




\begin{tabular}{|c|c|c|}
\hline Type of $G$ & $J$ & Type of $\mathscr{A}_{J}$ \\
\hline$A_{n}(n \geq 2)$ & $\{1\}$ & - \\
$A_{n}(n \geq 2)$ & $\{2\}$ & $A_{n-2}$ \\
$A_{n}(n \geq 2)$ & $\{1,2\}$ & $A_{n-1}$ \\
$A_{n}(n \geq 2)$ & $\{1, n\}$ & $\left(A_{1}\right)^{n-1}$ \\
$A_{n}(n \geq 3)$ & $\{1, n-1\}$ & $A_{2 n-4}$ \\
$A_{n}(n \geq 3)$ & $\{1,2, n\}$ & $A_{2 n-3}$ \\
\hline$A_{4}$ & $\{2,3\}$ & $D_{4}$ \\
$A_{4}$ & $\{1,2,3\}$ & $D_{5}$ \\
$A_{4}$ & $\{1,2,3,4\}$ & $D_{6}$ \\
\hline$A_{5}$ & $\{3\}$ & $D_{4}$ \\
$A_{5}$ & $\{1,3\}$ & $E_{6}$ \\
$A_{5}$ & $\{2,3\}$ & $E_{6}$ \\
$A_{5}$ & $\{1,2,3\}$ & $E_{7}$ \\
\hline$A_{6}$ & $\{3\}$ & $E_{6}$ \\
$A_{6}$ & $\{2,3\}$ & $E_{8}$ \\
\hline$A_{7}$ & $\{3\}$ & $E_{8}$ \\
\hline$D_{n}(n \geq 4)$ & $\{n\}$ & $\left(A_{1}\right)^{n-2}$ \\
\hline$D_{4}$ & $\{1,2\}$ & $A_{5}$ \\
\hline$D_{5}$ & $\{1\}$ & $A_{5}$ \\
\hline
\end{tabular}

Table 2: Algebras $\mathscr{A}_{J}$ of finite cluster type.

This implies that

$$
\Delta_{\varpi_{3}, \omega_{3}} \widetilde{\varphi_{M}(\lambda)}=\widetilde{\varphi_{S_{3}}} \widetilde{\varphi_{N_{1}}}-\widetilde{\varphi_{N_{2}}}-\Delta_{\varpi_{3}, \varpi_{3}}\left(\widetilde{\varphi_{L_{1}}}+\widetilde{\varphi_{L_{2}}}+\widetilde{\varphi_{L_{4}}}\right),
$$

which shows that $\widetilde{\varphi_{M(\lambda)}}$ belongs to the localization of $\widetilde{\mathscr{A}_{\{3\}}}$ with respect to $\Delta_{\varpi_{3}, \Phi_{3}}$. This finishes the proof of Conjecture 10.4 in type $D_{4}$.

\section{Finite type classification}

11.1 Using the explicit initial seed described in 9.3.3 it is possible to give a complete list of the algebras $\mathscr{A}_{J}$ which are of finite type as cluster algebras. The results are summarized in Table 2 , Here, we label the vertices of the Dynkin diagram of type $D_{n}$ in such a way that $L\left(\varpi_{1}\right)$ and $L\left(\varpi_{2}\right)$ are the two spin representations and $L\left(\varpi_{n}\right)$ is the vector representation. We have only listed one representative of each orbit under a diagram automorphism. For example, in type $A_{n}$ we have an order 2 diagram automorphism mapping $J=\{1,2\}$ to $J^{\prime}=\{n-1, n\}$. Clearly, $\mathscr{A}_{J^{\prime}}$ has the same cluster type as $J$, namely $A_{n-1}$.

11.2 The classification when $J=I$ (i.e. in the case of $\mathbb{C}[N]$ or $\mathbb{C}\left[B^{-} \backslash G\right]$ ) was given by Berenstein, Fomin and Zelevinsky [4]. The only finite type cases are $A_{n}(n \leqslant 4)$.

11.3 The classification when $J=\{j\}$ is a singleton and $G$ is of type $A_{n}$ (the Grassmannian $\operatorname{Gr}(j, n+1))$ was already known [32]. Note that when $J=\{1\}$ (the projective space $\mathbb{P}^{n}(\mathbb{C})$ ) the 


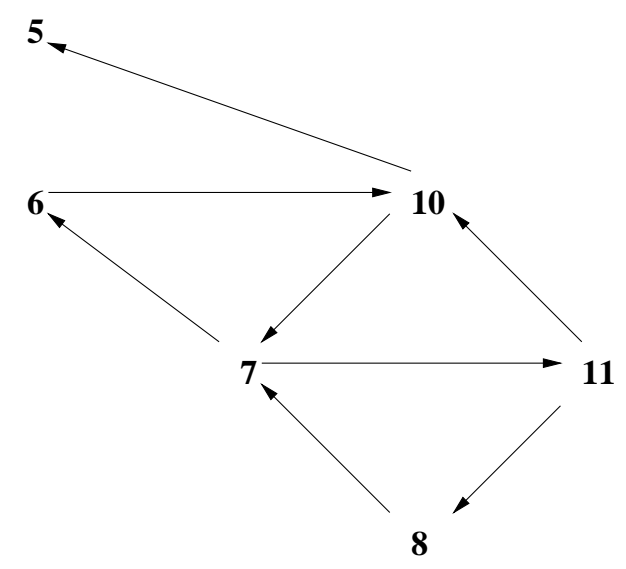

Figure 6: The graph $\Gamma_{\mathbf{i}, J}$ for $J=\{1,3\}$ in type $A_{5}$.

cluster structure is trivial. Indeed $\operatorname{Sub} Q_{\{1\}}$ has only $n$ indecomposable objects which are all Extprojective. Thus $\mathscr{A}_{\{1\}}$ (resp. $\widetilde{\mathscr{A}}_{\{1\}}$ ) has no cluster variable and is reduced to its coefficient ring, a polynomial ring in $n$ (resp. $n+1$ ) variables.

11.4 We now indicate how to obtain the classification.

11.4.1 The first step is to check that all cluster algebras $\mathscr{A}_{J}$ of Table 2 are indeed of finite type. For this, one chooses $\mathbf{i} \in R\left(w_{0}, K\right)$ and computes following 9.3 .3 the exchange matrix $B(\mathbf{i}, J)$. Let $\Gamma_{\mathbf{i}, J}$ denote the subgraph of $\Gamma_{\mathbf{i}}$ corresponding to the principal part of $B(\mathbf{i}, J)$. By [11], one then has to find a sequence of mutations transforming this graph into an orientation of the Dynkin diagram of the claimed cluster type. In each case, this is a straightforward verification. We illustrate it in the next example.

Example 11.1 We continue Example 9.5. Here $G$ is of type $A_{5}$ and $J=\{1,3\}$. The graph $\Gamma_{\mathbf{i}, J}$ is shown in Figure 6 If one performs a sequence of 3 mutations, first at vertex 6 , then at vertex 11 , finally at vertex 7 , one gets a quiver of type $E_{6}$, in agreement with Table 2 .

For the case $D_{n}, J=\{n\}$, see also Section 12 below. For the case $D_{5}, J=\{1\}$, see also Section 13 below.

11.4.2 One then needs to check a number of "minimal" infinite cases. In type $A$ these are

- $A_{5}: J=\{1,3,5\}$,

- $A_{6}: J=\{1,3\},\{1,4\},\{3,4\}$,

- $A_{7}: J=\{2,3\},\{3,7\}$,

- $A_{n}(n \geq 5): J=\{2, n-1\}$.

In type $D$ these are

- $D_{4}: J=\{1,2,4\}$,

- $D_{5}: J=\{1,2\},\{1,5\}$, 


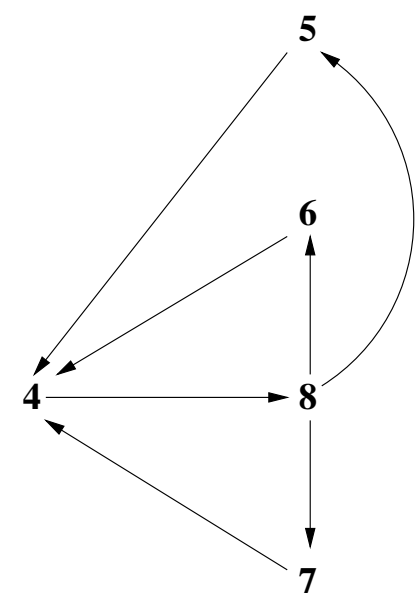

Figure 7: The graph $\Gamma_{\mathbf{i}, J}$ for $J=\{3\}$ in type $D_{4}$.

- $D_{6}: J=\{1\}$,

- $D_{n}(n \geq 4): J=\{n-1\}$.

In type $E$, labelling the Dynkin diagrams as in [6], these are

- $E_{6}: J=\{1\},\{2\}$.

- $E_{7}: J=\{7\}$.

- $E_{8}: J=\{8\}$.

These cases are settled by calculating as in 11.4.1 the graph $\Gamma_{\mathbf{i}, J}$. Then, one may either check that $\Gamma_{\mathbf{i}, J}$ contains a full subgraph from the list of minimal infinite subgraphs of Seven [33], or find a sequence of mutations transforming $\Gamma_{\mathbf{i}, J}$ into a graph (containing a full subgraph) of affine type.

Example 11.2 Take $G$ of type $D_{4}$ and $J=\{3\}$. Then

$$
\mathbf{i}=(1,2,4,3,1,2,4,3,1,2,4,3)
$$

belongs to $R\left(w_{0}, K\right)$. The graph $\Gamma_{\mathbf{i}, J}$ is shown in Figure 7. If one performs a mutation at vertex 4, one gets a quiver of affine type $D_{4}$.

For the case $D_{6}, J=\{1\}$, see also Section 13 below.

11.4.3 To prove that there are no other finite type cluster algebras $\mathscr{A}_{J}$ than those listed in Table 2 , one uses the following obvious

Lemma 11.3 (i) Let $\mathscr{A}_{J}$ and $\mathscr{A}_{J^{\prime}}$ be two algebras attached to the same Dynkin diagram and suppose that $J \subset J^{\prime}$. If $\mathscr{A}_{J}$ has infinite cluster type then $\mathscr{A}_{J^{\prime}}$ has infinite cluster type.

(ii) Let $\mathscr{A}_{J}$ and $\mathscr{A}_{J}^{\prime}$ be two algebras attached to two Dynkin diagrams $\Delta$ and $\Delta^{\prime}$ and suppose that $\Delta$ is a full subdiagram of $\Delta^{\prime}$. If $\mathscr{A}_{J}$ has infinite cluster type then $\mathscr{A}_{J}^{\prime}$ has infinite cluster type. 
11.4.4 Assume that $G$ is of type $A_{n}$. By [32] we know that if $n \geq 8$ the only cluster algebras $\mathscr{A}_{\{j\}}$ of finite type are obtained for $j=1,2, n-1, n$. It follows from Lemma 11.3 (i) that for $n \geq 8$ the algebra $\mathscr{A}_{J}$ can have finite cluster type only if $J \subseteq\{1,2, n-1, n\}$. Since $J=\{2, n-1\}$ yields an infinite type by 11.4.2, this is also the case for $J=\{1,2, n-1\}$ and $J=\{1,2, n-1, n\}$. Hence, the only cases left for $n \geq 8$ are those of Table 2 . For $A_{7}$, we need to check that no $J$ of the form $\{j, 3\}$ gives a finite type. By 11.4.2, $\{2,3\}$ and $\{3,7\}$ can be excluded. Using Lemma 11.3 (ii) we can also exclude $\{1,3\}$ and $\{2,3\}$ since they already give an infinite type for $A_{6}$. For $\{3,5\}$ and $\{3,6\}$, we use again Lemma 11.3 (ii) and restrict to type $A_{6}$ by removing the vertex 1 of the Dynkin diagram. This yields $J=\{2,4\}$ and $J=\{2,5\}$ in type $A_{6}$. These can be ruled out by using 11.4.2 again $\left(J=\{2,4\}\right.$ gives already an infinite type for $\left.A_{5}\right)$. This finishes type $A_{7}$. The types $A_{6}$ and $A_{5}$ are dealt with similarly. Details are omitted. This finishes the classification in type $A$.

11.4.5 Assume that $G$ is of type $D_{n}$. If $n \geq 6$ and $j \neq n$ then $\mathscr{A}_{\{j\}}$ has infinite type. This is easily shown by induction on $n$. Indeed if $n=6$, by 11.4.2 we can exclude $j=1,2,5$. By Lemma 11.3(ii) and using again 11.4.2 for $D_{5}$ and $D_{4}$ we can exclude $j=4$ and $j=3$. Thus $n=6$ is checked. If the claim holds for $D_{n-1}$ then we can exclude $j=n-1$ by 11.4.2 and use Lemma11.3(ii) and the assumption for $D_{n-1}$ for all other $j$ 's. If $n=5$, and $\mathscr{A}_{J}$ is of finite type, we must have $J \subseteq\{1,2,5\}$. By 11.4.2 we can exclude all pairs in $\{1,2,5\}$, so we are left with $J=\{1\}$ or $J=\{5\}$ in agreement with Table $2(J=\{2\}$ and $J=\{1\}$ are conjugate under a diagram automorphism). If $n=4$, we have a diagram automorphism of order 3 exchanging 1, 2 and 4. Taking into account 11.4.2, we see that we are left with the cases of Table 2 (up to isomorphism). This finishes the classification in type $D$.

11.4.6 Assume that $G$ is of type $E_{6}$. We have a diagram automorphism exchanging 1 and 6 . Thus, using 11.4.2 we see that $\mathscr{A}_{\{j\}}$ is of infinite type for $j=1,2,6$. Using Lemma11.3 (ii) and 11.4.5. we obtain by reduction to $D_{5}$ that $\mathscr{A}_{\{j\}}$ is also of infinite type for $j=3,4,5$. Thus $\mathscr{A}_{J}$ is infinite for every $J$. The cases when $G$ is of type $E_{7}$ or $E_{8}$ follow by means of Lemma11.3 (ii) and 11.4.2. This finishes the classification in type $E$.

11.5 When $\mathscr{A}_{J}$ has infinite cluster type, it has an infinite number of cluster variables. Therefore the category $\operatorname{Sub} Q_{J}$ has an infinite number of indecomposable rigid modules.

When $\mathscr{A}_{J}$ has finite cluster type, one can knit the Auslander-Reiten quiver of $\operatorname{Sub} Q_{J}$. One obtains a finite connected quiver drawn either on a cylinder or on a Möbius band. The corresponding stable Auslander-Reiten quiver, obtained by deleting the Ext-projective modules, is isomorphic to the Auslander-Reiten quiver of the cluster category of the same type as $\mathscr{A}_{J}$, introduced by Buan, Marsh, Reineke, Reiten and Todorov [8].

Example 11.4 We continue Examples 9.5 and 11.1. Here $G$ is of type $A_{5}$ and $J=\{1,3\}$. The Auslander-Reiten quiver of $\operatorname{Sub} Q_{J}$ is shown in Figure 8 This quiver is drawn on a Möbius band. The horizontal dashed lines at the top and at the bottom are to be identified after performing a halfturn. The relative Auslander-Reiten translation $\tau_{J}$ is going up. Each indecomposable $\Lambda$-module $M$ has a grading with semisimple homogeneous components. We represent $M$ by displaying the layers of this filtration. Thus,

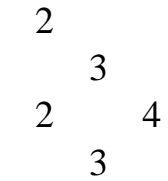




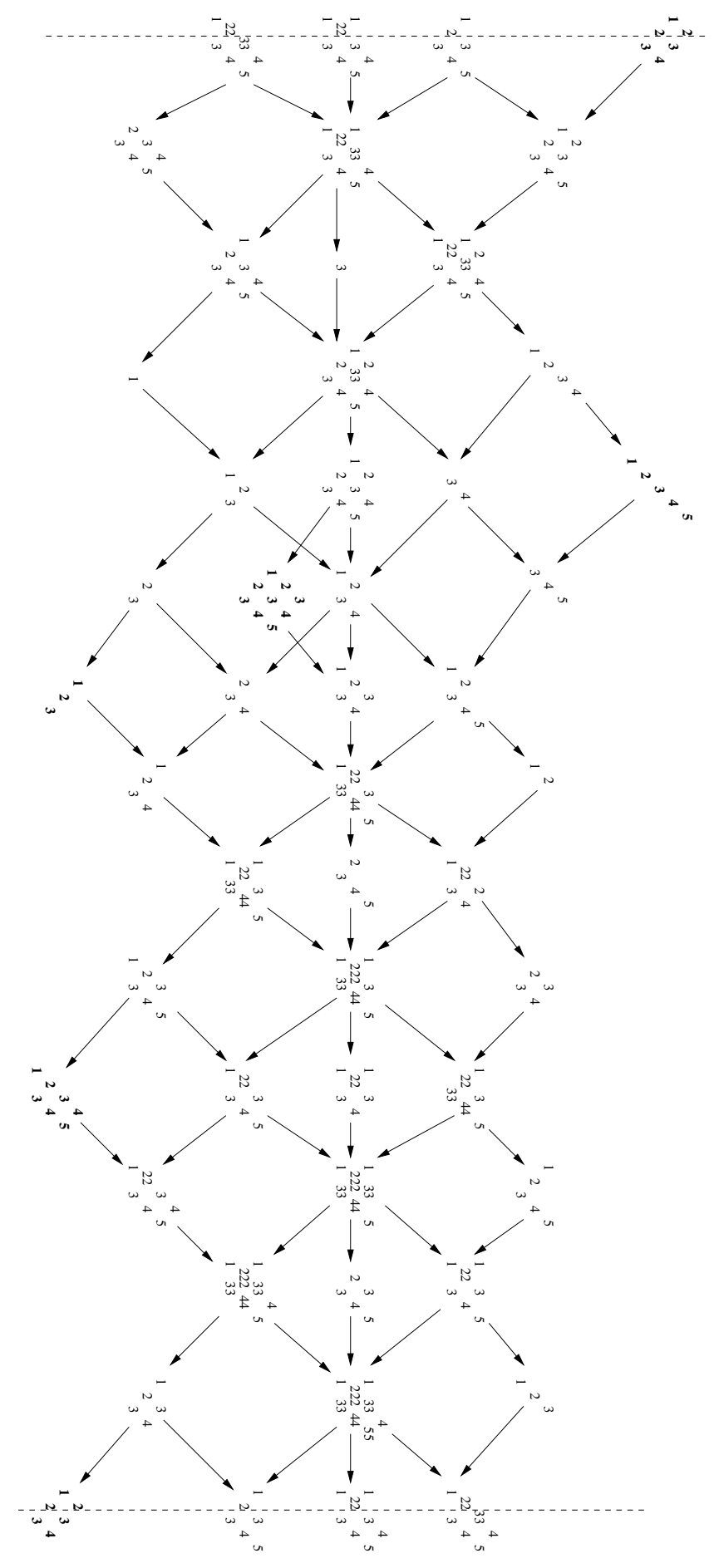

Figure 8: The Auslander-Reiten quiver for $J=\{1,3\}$ in type $A_{5}$.

stands for a 6-dimensional module with graded components $S_{2}, S_{1} \oplus S_{3}, S_{2} \oplus S_{4}$ and $S_{3}$, from top to bottom. To determine this quiver, we have first calculated the $\tau_{J}$-orbits using Proposition 3.4, which states that $\tau_{J}^{-1}$ is equal to the relative syzygy functor. The five Ext-projective modules $L_{i}$ are printed in bold type. If one erases these five vertices as well as the corresponding arrows, 
one obtains the stable Auslander-Reiten quiver of $\operatorname{Sub} Q_{J}$, which is isomorphic to the quiver of a cluster category of type $E_{6}$.

\section{The coordinate ring of a smooth quadric in $\mathbb{P}^{2 n-1}(\mathbb{C})$}

When $G$ is of type $D_{n}$ and $J=\{n\}$, the construction of the previous sections yields a cluster algebra structure on the homogeneous coordinate ring of a smooth quadric in $\mathbb{P}^{2 n-1}(\mathbb{C})$, and a finite type subcategory $\operatorname{Sub} Q_{n}$ of $\bmod \Lambda$ which can be regarded as a lift of this ring. We shall present this example in some detail.

12.1 Let $n \geq 4$. Let $U=\mathbb{C}^{2 n}$ and $\mathbb{P}=\mathbb{P}(U)=\mathbb{P}^{2 n-1}(\mathbb{C})$. Let $\left(u_{1}, \ldots, u_{2 n}\right)$ be a fixed basis of $U$ and $\left(y_{1}, \ldots, y_{2 n}\right)$ the coordinate functions with respect to this basis. We denote by $\left[y_{1}: y_{2}: \cdots: y_{2 n}\right]$ the corresponding system of homogeneous coordinates on $\mathbb{P}$ and we consider the smooth quadric $\mathscr{Q}$ in $\mathbb{P}$ given by the equation

$$
q\left(y_{1}, \ldots, y_{2 n}\right):=\sum_{i=1}^{n}(-1)^{i} y_{i} y_{2 n+1-i}=0 .
$$

In other words, $\mathscr{Q}$ is isomorphic to the variety of isotropic lines in the quadratic space $(U, q)$. Note that every smooth quadric in $\mathbb{P}$ can be brought to equation (11) by an appropriate change of coordinates. The homogeneous coordinate ring of $\mathscr{Q}$ is

$$
\mathbb{C}[\mathscr{Q}]=\mathbb{C}\left[y_{1}, \ldots, y_{2 n}\right] /\left(q\left(y_{1}, \ldots, y_{2 n}\right)=0\right) .
$$

12.2 Let $G$ be the group of linear transformations of $U$ preserving the quadratic form $q$. We regard $G$ as acting on the right on $U$, i.e. elements of $U$ are regarded as row vectors. We identify $g \in G$ with its matrix with respect to $\left(u_{1}, \ldots, u_{2 n}\right)$ : the $i$ th row of the matrix $g$ is the list of coordinates of $u_{i} g$. The group $G=S O_{2 n}(\mathbb{C})$ is a group of type $D_{n}$. In this realization, the subgroup of diagonal matrices of $G$ is a maximal torus, and the subgroup of upper (resp. lower) triangular matrices of $G$ is a Borel subgroup denoted by $B\left(\right.$ resp. $\left.B^{-}\right)$. We label the vertices of the Dynkin diagram in such a way that 1 and 2 correspond to the two spin representations and $n$ to the vector representation $U$.

12.3 Let $J=\{n\}$ and $K=\{1,2, \ldots, n-1\}$. The quadric $\mathscr{Q}$ is isomorphic to $B_{K}^{-} \backslash G$. Indeed, $\mathbb{C} u_{1}$ is an isotropic line and $\mathscr{Q}$ is the $G$-orbit of $\mathbb{C} u_{1}$, the stabilizer of $\mathbb{C} u_{1}$ being equal to $B_{K}^{-}$. Therefore, for $g \in G$, the coordinates of the vector $u_{1} g$ give a system of homogeneous coordinates for the point $\mathbb{C} u_{1} g \in \mathscr{Q}$, that we can and shall continue to denote by $y_{1}, \ldots, y_{2 n}$. The affine open subset given by the non-vanishing of $y_{1}$ is isomorphic to $N_{K}$. Thus, setting $z_{k}=y_{k} / y_{1}$

$$
\mathbb{C}\left[N_{K}\right]=\mathbb{C}\left[z_{2}, \ldots, z_{2 n}\right] /\left(q\left(1, z_{2}, \ldots, z_{2 n}\right)=0\right) .
$$

12.4 The functions on $G$ mapping $g$ to the coordinates of the vector $u_{1} g$ are regular, and are nothing else than the generalized minors $\Delta_{\varpi_{n}, v\left(\varpi_{n}\right)}$ for $v$ in the Weyl group. In particular the coordinate of $u_{1}$ in $u_{1} g$ is equal to $\Delta_{\varpi_{n}, \Phi_{n}}(g)$, and the coordinate of $u_{2 n}$ in $u_{1} g$ is equal to $\Delta_{\varpi_{n}, w_{0}\left(\varpi_{n}\right)}(g)$. The restrictions $D_{\Phi_{n}, v\left(\varpi_{n}\right)}$ of all these functions to the unipotent radical $N$ of $B$ are the elements $\varphi_{M}$ of $\mathbb{C}[N]$, where $M$ runs over the $2 n$ submodules $M$ of the injective $\Lambda$-module $Q_{n}$. In particular

$$
D_{\bar{\omega}_{n}, \bar{\omega}_{n}}=1=\varphi_{0}=z_{1}, \quad D_{\bar{\omega}_{n}, w_{0}\left(\varpi_{n}\right)}=\varphi_{Q_{n}}=z_{2 n},
$$

where $\mathbf{0}$ denotes the trivial submodule of $Q_{n}$. 
12.5 The category $\operatorname{Sub} Q_{n}$ has a finite number of indecomposable objects, which are all rigid. Here is a complete list:

- the $2 n-1$ nonzero submodules of $Q_{n}$, with pairwise distinct dimension vectors:

$$
\begin{aligned}
& {[0, \ldots, 0,1],[0, \ldots, 0,1,1], \ldots,[0,1, \ldots, 1,1],} \\
& {[1,0,1, \ldots, 1,1],[1,1,1, \ldots, 1,1],[1,1,2,1, \ldots, 1,1],} \\
& {[1,1,2,2,1, \ldots, 1,1], \ldots,[1,1,2,2, \ldots, 2] .}
\end{aligned}
$$

The modules with dimension vectors $[0,1, \ldots, 1,1],[1,0,1, \ldots, 1,1],[1,1,2,2, \ldots, 2]$ are the relatively projective modules $L_{2}, L_{1}, L_{n}=Q_{n}$, respectively. We shall denote the regular functions on $N$ corresponding to these $2 n-1$ modules by $z_{2}, \ldots, z_{2 n}$, in agreement with 12.3 and 12.4 They generate $\mathbb{C}\left[N_{K}\right]$ and satisfy $q\left(1, z_{2}, \ldots, z_{2 n}\right)=0$.

- the $n-3$ indecomposable submodules of $Q_{n} \oplus Q_{n}$ with socle $S_{n} \oplus S_{n}$ (up to isomorphism). They all have the same dimension vector as $Q_{n}$, namely $[1,1,2,2, \ldots, 2]$, and they all are projective objects in $\operatorname{Sub} Q_{n}$. They form the remaining indecomposable projectives $L_{3}, \ldots, L_{n-1}$ of $\operatorname{Sub} Q_{n}$. We denote the corresponding functions on $N$ by $p_{3}, \ldots, p_{n-1}$. One can check that for $3 \leqslant k \leqslant n-1$,

$$
p_{k}=z_{n+1-k} z_{n+k}-z_{n-k} z_{n+k+1}+\cdots+(-1)^{n-k-1} z_{2} z_{2 n-1}+(-1)^{n-k} z_{2 n} .
$$

One can also check that $p_{k}=D_{u_{k}\left(\varpi_{k}\right), w_{0}\left(\varpi_{k}\right)}$ for some appropriate element $u_{k}$ of $W$.

As an illustration, the Auslander-Reiten quiver of the category $\operatorname{Sub} Q_{5}$ in type $D_{5}$ is displayed in Figure 9 The quiver is drawn on a cylinder, obtained by identifying the two vertical dashed lines. The 5 projective objects are written in bold type.

12.6 The cluster algebra structure $\mathscr{A}_{J}$ on $\mathbb{C}\left[N_{K}\right]$ is obtained as follows. The coefficient ring is generated by $p_{1}, \ldots, p_{n}$, where $p_{1}:=z_{n}, p_{2}:=z_{n+1}, p_{n}:=z_{2 n}$ and the remaining $p_{k}$ 's are defined above. There are $2(n-2)$ cluster variables, namely

$$
z_{2}, z_{3}, \ldots, z_{n-1}, z_{n+2}, \ldots, z_{2 n-1}
$$

The first $n-2$ variables form a cluster and for $2 \leqslant k \leqslant n-1$ we have the exchange relations

$$
z_{k} z_{2 n-k+1}= \begin{cases}p_{n-k+1}+p_{n-k+2} & \text { if } 2 \leqslant k \leqslant n-2 \\ p_{1} p_{2}+p_{3} & \text { if } k=n-1 .\end{cases}
$$

This shows that $\mathscr{A}_{J}=\mathbb{C}\left[N_{K}\right]$ has finite cluster type equal to $\left(A_{1}\right)^{n-2}$, in agreement with Table 2 ,

12.7 The cluster algebra structure $\widetilde{\mathscr{A}_{J}}$ on $\mathbb{C}[\mathscr{Q}]$ is obtained as follows. The coefficient ring is generated by $q_{0}, q_{1}, \ldots, q_{n}$, where $q_{0}:=y_{1}, q_{1}:=y_{n}, q_{2}:=y_{n+1}, q_{n}:=y_{2 n}$ and for $3 \leqslant k \leqslant n-1$,

$$
q_{k}:=y_{n+1-k} y_{n+k}-y_{n-k} y_{n+k+1}+\cdots+(-1)^{n-k-1} y_{2} y_{2 n-1}+(-1)^{n-k} y_{1} y_{2 n} .
$$

There are $2(n-2)$ cluster variables, namely

$$
y_{2}, y_{3}, \ldots, y_{n-1}, y_{n+2}, \ldots, y_{2 n-1} \text {. }
$$




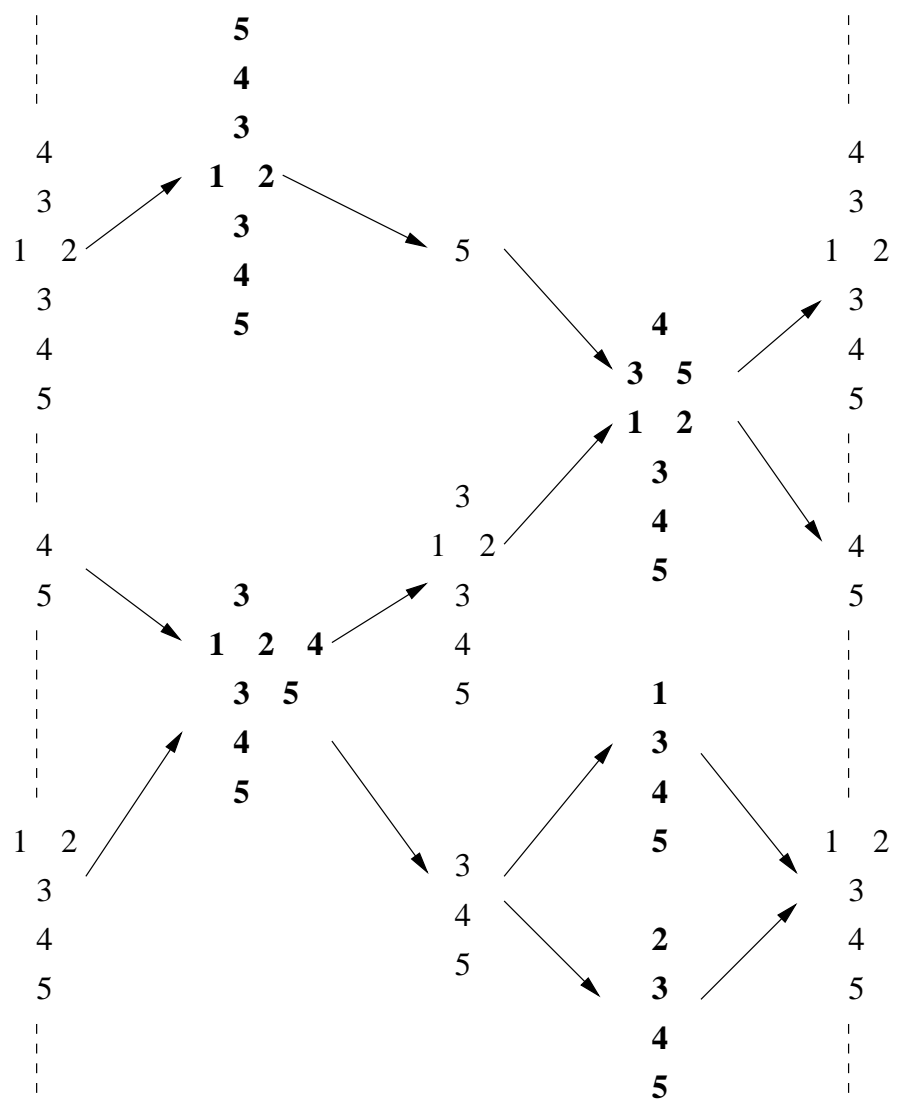

Figure 9: The Auslander-Reiten quiver of the category $\operatorname{Sub} Q_{5}$ in type $D_{5}$.

The first $n-2$ form a cluster and for $2 \leqslant k \leqslant n-1$ we have the exchange relations

$$
y_{k} y_{2 n-k+1}= \begin{cases}q_{n-1}+q_{0} q_{n} & \text { if } k=2, \\ q_{n-k+1}+q_{n-k+2} & \text { if } 3 \leqslant k \leqslant n-2, \\ q_{1} q_{2}+q_{3} & \text { if } k=n-1 .\end{cases}
$$

Thus, $\widetilde{\mathscr{A}_{J}}=\mathbb{C}[\mathscr{Q}]$ is also a cluster algebra of type $\left(A_{1}\right)^{n-2}$.

12.8 When $n=3, \mathbb{C}[\mathscr{Q}]$ has a cluster algebra structure of type $A_{1}$. Using the same notation as above for the generators $y_{1}, \ldots, y_{6}$ and the coefficients $q_{0}, \ldots, q_{3}$, the unique exchange relation reads

$$
y_{2} y_{5}=q_{1} q_{2}+q_{0} q_{3} .
$$

Note that $D_{3} \cong A_{3}$ and that $\mathscr{Q}$ is isomorphic to the Grassmannian of 2-planes in $\mathbb{C}^{4}$.

\section{Isotropic Grassmannians}

We retain the notation of Section 12 Let $\mathscr{G}$ denote the Grassmann variety of totally isotropic $n$-subspaces of $U$. This variety has two connected components, and we shall denote by $\mathscr{G}_{0}$ the component containing the subspace spanned by $\left(u_{1}, \ldots, u_{n}\right)$. The stabilizer of this subspace for 


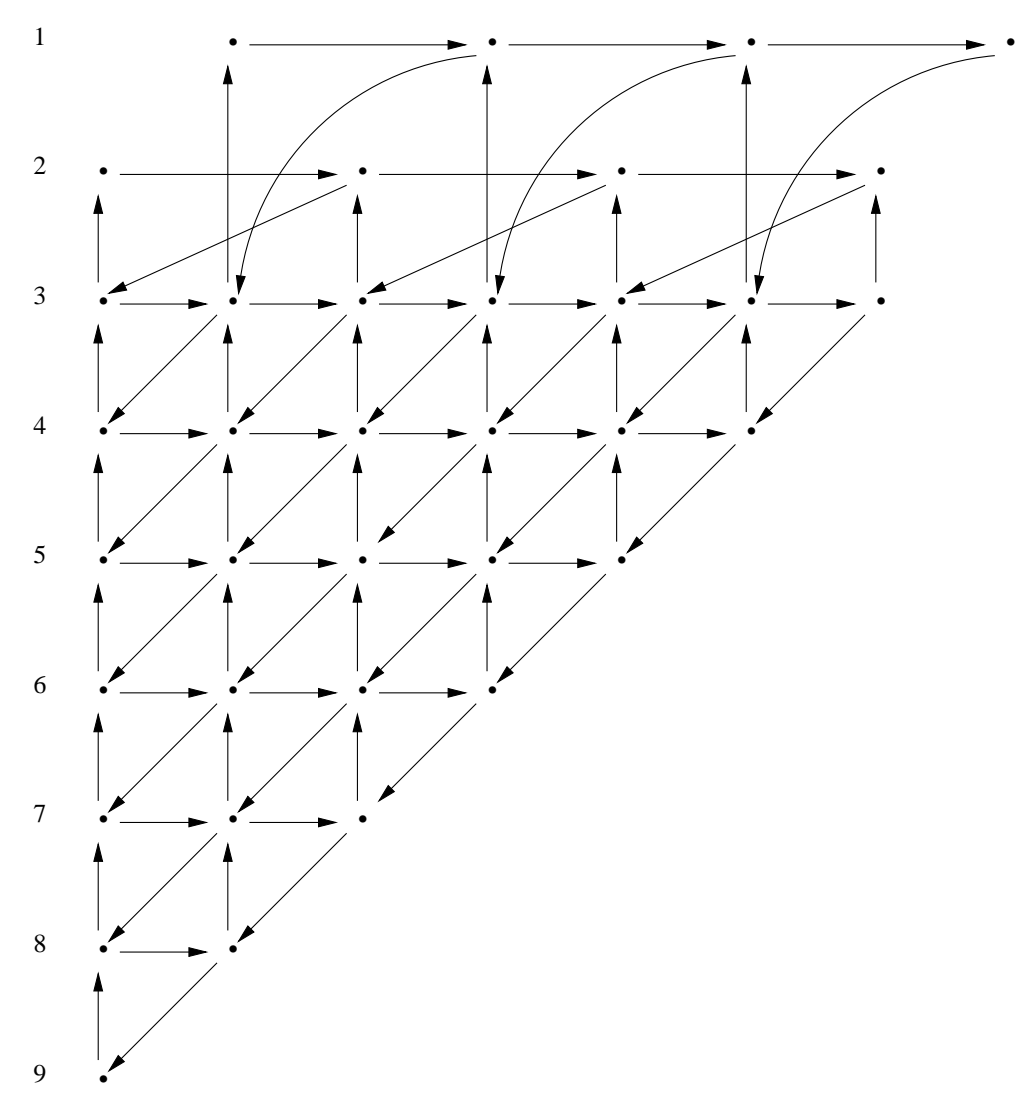

Figure 10: The triangular grid for $D_{9}$.

the natural action of $G$ is the maximal parabolic subgroup $B_{K}^{-}$, where now $K=\{2, \ldots, n\}$, that is, $J=\{1\}$. Thus $\mathscr{G}_{0}$ is isomorphic to $B_{K}^{-} \backslash G$. We shall now discuss the cluster algebra structure on $\mathbb{C}\left[\mathscr{G}_{0}\right]$ and the corresponding subcategory $\operatorname{Sub} Q_{1}$.

13.1 As in 10.3 .1 the rule of 9.3 .3 gives us a unique initial cluster for $\mathscr{A}_{\{1\}}$. The graph encoding the exchange matrix of this cluster has the shape of a triangular grid in which the first two rows, corresponding to the exceptional vertices 1 and 2 of the Dynkin diagram of $D_{n}$, have a special structure. A typical example $(n=9)$ is displayed in Figure 10 (compare Figure 2, the rectangular grid attached to the ordinary Grassmannian). The $n$ generators of the coefficient ring correspond to the leftmost vertices on each row. It is easy to see that this graph yields a cluster algebra of infinite type, except for $n=4$ and $n=5$.

13.2 When $n=4$, because of the order 3 symmetry of the Dynkin diagram, $\mathscr{G}_{0}$ is isomorphic to the quadric $\mathscr{Q}$ of Section 12 , and $\operatorname{Sub} Q_{1}$ is equivalent to $\operatorname{Sub} Q_{4}$. In particular $\widetilde{\mathscr{A}}_{\{1\}}=\mathbb{C}\left[\mathscr{G}_{0}\right]$ is a cluster algebra of type $A_{1} \times A_{1}$.

13.3 When $n=5$, Sub $Q_{1}$ is a category of finite type with 25 indecomposable objects (up to isomorphism), 5 of them being Ext-projective. The Auslander-Reiten quiver of $\operatorname{Sub} Q_{1}$ is displayed in Figure 11 It is drawn on a Möbius band obtained by identifying the two vertical dashed lines after performing a half-turn. The stable Auslander-Reiten quiver (obtained by deleting the 5 projectives) is the quiver of a cluster category of type $A_{5}$, in agreement with Table 2 . 


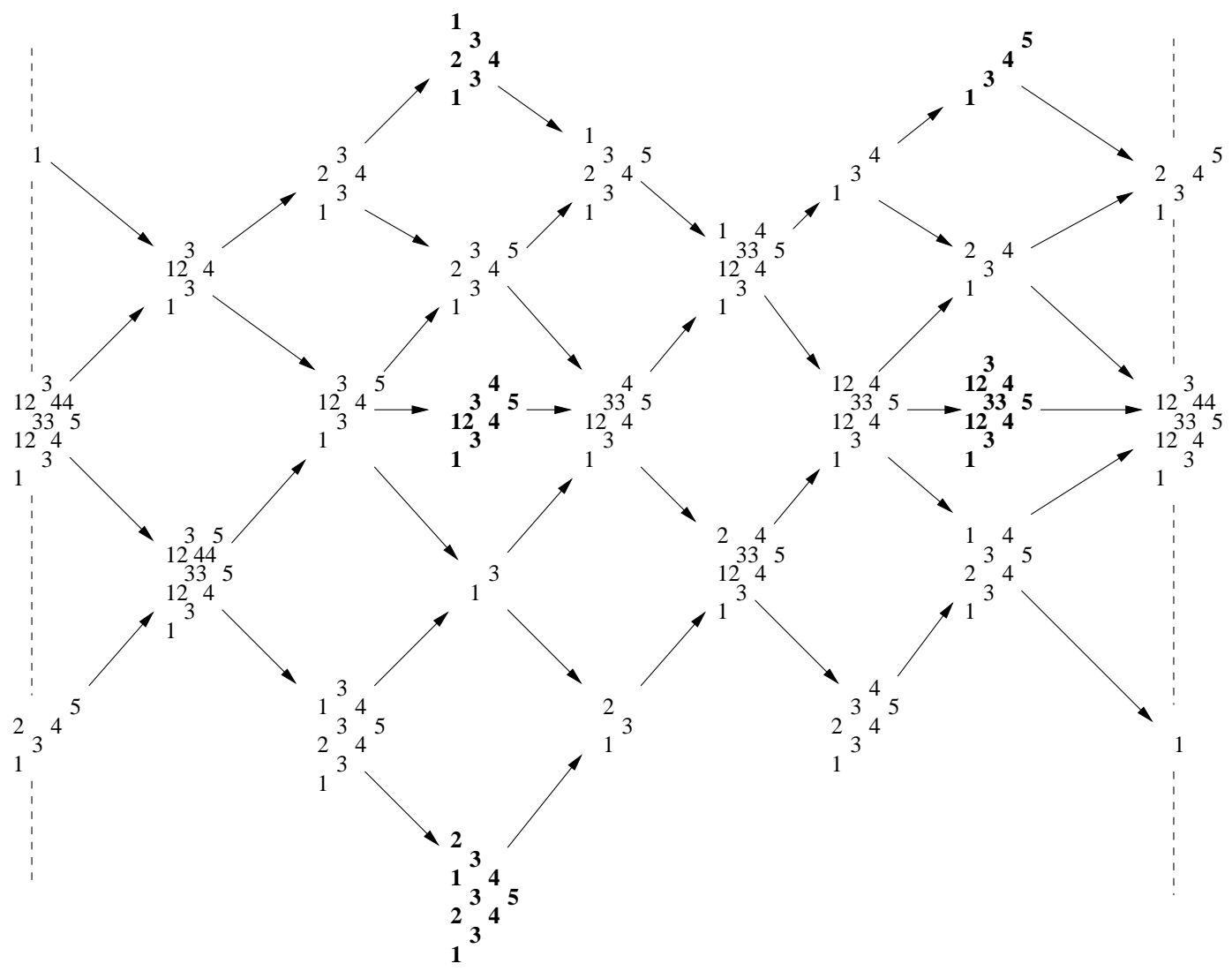

Figure 11: The Auslander-Reiten quiver of the category $\operatorname{Sub} Q_{1}$ in type $D_{5}$.

We shall now describe the cluster algebra structure $\widetilde{\mathscr{A}}_{\{1\}}$ on $\mathbb{C}\left[\mathscr{G}_{0}\right]$. First we express the cluster variables and the generators of the coefficient ring in terms of the generalized minors $\Delta_{\bar{\omega}_{1}, u\left(\varpi_{1}\right)}$ which generate $\mathbb{C}\left[\mathscr{G}_{0}\right]$. (Note that, strictly speaking, these minors are not functions on $G=S_{2 n}(\mathbb{C})$ but only on the corresponding simply connected group $\operatorname{Spin}_{2 n}(\mathbb{C})$.) The 16 minors $\Delta_{\varpi_{1}, u\left(\varpi_{1}\right)}$ will be denoted for short by $\Delta_{i}(1 \leqslant i \leqslant 16)$ according to Table 3 Among them,

$$
\Delta_{1}=q_{0}, \quad \Delta_{7}=\widetilde{\varphi_{L_{5}}}=q_{5}, \quad \Delta_{10}=\widetilde{\varphi_{L_{1}}}=q_{1}, \quad \Delta_{16}=\widetilde{\varphi_{L_{2}}}=\widetilde{\varphi_{Q_{1}}}=q_{2}
$$

are generators of the coefficient ring of $\widetilde{\mathscr{A}_{\{1\}}}$. The two other generators of the coefficent ring are

$$
q_{3}=\widetilde{\varphi_{L_{3}}}=\Delta_{4} \Delta_{15}-\Delta_{3} \Delta_{16}, \quad q_{4}=\widetilde{\varphi_{L_{4}}}=\Delta_{2} \Delta_{14}-\Delta_{1} \Delta_{13}
$$

The initial cluster, obtained by lifting to $\widetilde{\mathscr{A}_{\{1\}}}$ the cluster of 13.1 consists of the functions

$$
z_{1}=\Delta_{2}, \quad z_{2}=\Delta_{3}, \quad z_{3}=\Delta_{4}, \quad z_{4}=\Delta_{5}, \quad z_{5}=\Delta_{2} \Delta_{8}-\Delta_{1} \Delta_{10} .
$$




\begin{tabular}{|c|c|c|}
\hline$i$ & $u$ & mutation sequence for $\Delta_{i}$ \\
\hline 1 & $e$ & $q_{0}$ \\
2 & $s_{1}$ & $z_{1}$ \\
3 & $s_{3} s_{1}$ & $z_{2}$ \\
4 & $s_{2} s_{3} s_{1}$ & $z_{3}$ \\
5 & $s_{4} s_{3} s_{1}$ & $z_{4}$ \\
6 & $s_{2} s_{4} s_{3} s_{1}$ & $\mu_{2}$ \\
7 & $s_{5} s_{4} s_{3} s_{1}$ & $q_{5}$ \\
8 & $s_{3} s_{2} s_{4} s_{3} s_{1}$ & $\mu_{1}$ \\
9 & $s_{2} s_{5} s_{4} s_{3} s_{1}$ & $\mu_{2} \mu_{1} \mu_{5} \mu_{4}$ \\
10 & $s_{1} s_{3} s_{2} s_{4} s_{3} s_{1}$ & $q_{1}$ \\
11 & $s_{5} s_{3} s_{2} s_{4} s_{3} s_{1}$ & $\mu_{1} \mu_{5} \mu_{4}$ \\
12 & $s_{1} s_{5} s_{3} s_{2} s_{4} s_{3} s_{1}$ & $\mu_{5} \mu_{4}$ \\
13 & $s_{4} s_{5} s_{3} s_{2} s_{4} s_{3} s_{1}$ & $\mu_{4} \mu_{3} \mu_{2} \mu_{1} \mu_{5} \mu_{4}$ \\
14 & $s_{1} s_{4} s_{5} s_{3} s_{2} s_{4} s_{3} s_{1}$ & $\mu_{1} \mu_{4} \mu_{3} \mu_{2} \mu_{1} \mu_{5} \mu_{4}$ \\
15 & $s_{3} s_{1} s_{4} s_{5} s_{3} s_{2} s_{4} s_{3} s_{1}$ & $\mu_{3} \mu_{1} \mu_{5} \mu_{4}$ \\
16 & $s_{2} s_{3} s_{1} s_{4} s_{5} s_{3} s_{2} s_{4} s_{3} s_{1}$ & $q_{2}$ \\
\hline
\end{tabular}

Table 3: Generalized minors $\Delta_{\bar{\omega}_{1}, u\left(\varpi_{1}\right)}$ in type $D_{5}$.

The exchange matrix of this cluster is

$$
\widetilde{B}=\left(\begin{array}{ccccc}
0 & 0 & 0 & 0 & 1 \\
0 & 0 & 1 & 1 & -1 \\
0 & -1 & 0 & 0 & 0 \\
0 & -1 & 0 & 0 & 1 \\
-1 & 1 & 0 & -1 & 0 \\
\hline 1 & 0 & 0 & 0 & -1 \\
0 & 0 & 1 & 0 & 0 \\
0 & 0 & -1 & 0 & 1 \\
0 & 0 & 0 & 1 & -1 \\
0 & 0 & 0 & -1 & 0 \\
\hline 1 & 0 & 0 & 0 & 0
\end{array}\right),
$$

where the successive rows are labelled by $z_{1}, z_{2}, z_{3}, z_{4}, z_{5}, q_{1}, q_{2}, q_{3}, q_{4}, q_{5}, q_{0}$. The last column of Table 3 indicates which sequence of mutations produces, starting from this initial cluster, each minor $\Delta_{i}$. This shows that $\widetilde{\mathscr{A}}_{\{1\}}$ contains a set of generators of $\mathbb{C}\left[\mathscr{G}_{0}\right]$. Hence, Conjecture 9.6 and Conjecture 10.4 are also proved in this case. (Note that $\varpi_{1}$ is a minuscule weight, so no localization is needed in Conjecture 10.4)

The remaining cluster variables all have degree $2 \varpi_{1}$ and are given by the following quadratic expressions in the minors $\Delta_{i}$ :

$$
\begin{gathered}
\Delta_{3} \Delta_{13}-\Delta_{1} \Delta_{15}, \quad \Delta_{4} \Delta_{14}-\Delta_{2} \Delta_{16}, \quad \Delta_{6} \Delta_{15}-\Delta_{5} \Delta_{16}, \quad \Delta_{2} \Delta_{11}-\Delta_{1} \Delta_{12}, \\
\Delta_{4} \Delta_{13}-\Delta_{1} \Delta_{16}, \quad \Delta_{9} \Delta_{15}-\Delta_{7} \Delta_{16}, \quad \Delta_{5} \Delta_{12}-\Delta_{7} \Delta_{10} .
\end{gathered}
$$

As already mentioned, $\widetilde{\mathscr{A}_{\{1\}}}=\mathbb{C}\left[\mathscr{G}_{0}\right]$ is a cluster algebra of finite type $A_{5}$. 


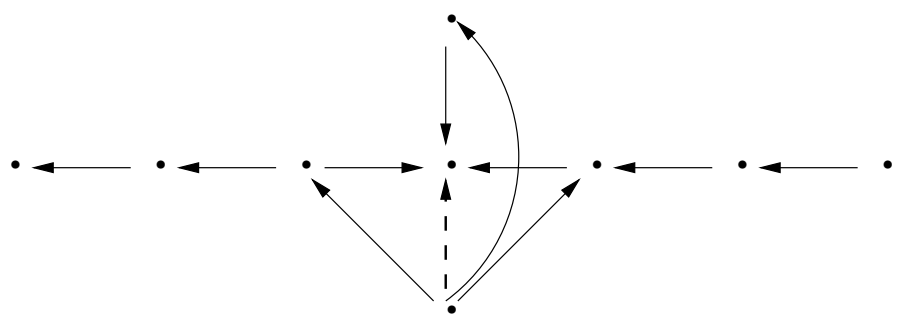

Figure 12: The elliptic diagram of type $E_{7}^{(1,1)}$.

13.4 When $n=6$, there exists a sequence of 6 mutations transforming the principal part of the triangular grid into the graph displayed in Figure 12. Here the dashed arrow stands for a pair of entries \pm 2 in the exchange matrix. Following [12], we propose to attach to this infinite type cluster algebra $\widetilde{\mathscr{A}_{\{1\}}}$ the elliptic type $E_{7}^{(1,1)}$.

\section{Remarks on the non simply-laced case}

14.1 Although the definition of the cluster algebra $\mathscr{A}_{J}$ was obtained using the representation theory of preprojective algebras, the definition of the initial seed in 9.3 .3 can be formulated without any reference to preprojective algebras. As a result, the same definition can serve to introduce similar cluster algebras $\mathscr{A}_{J}$ in the non simply-laced types. (This was suggested to us by Andrei Zelevinsky.) One can expect that $\mathscr{A}_{J}$ is again equal to $\mathbb{C}\left[N_{K}\right]$ and can be lifted to a cluster algebra structure $\mathscr{\mathscr { A }}_{J}$ on $\mathbb{C}\left[B_{K}^{-} \backslash G\right]$, where $G$ is now the corresponding algebraic group of non simply-laced type.

14.2 A similar study as in Section 11 gives the classification of all finite type cluster algebras $\mathscr{A}_{J}$ in the non simply-laced case. The results are summarized in Table 4 Our convention for labelling the Dynkin diagrams of type $B_{n}$ and $C_{n}$ is that the vertex associated with the vector representation is numbered $n$.

\begin{tabular}{|c|c|c|}
\hline Type of $G$ & $J$ & Type of $\mathscr{A}_{J}$ \\
\hline$B_{n}(n \geq 2)$ & $\{n\}$ & $\left(A_{1}\right)^{n-1}$ \\
\hline$C_{n}(n \geq 2)$ & $\{n\}$ & $\left(A_{1}\right)^{n-1}$ \\
\hline$B_{2}=C_{2}$ & $\{1,2\}$ & $B_{2}=C_{2}$ \\
\hline$B_{3}$ & $\{1\}$ & $C_{3}$ \\
\hline$C_{3}$ & $\{1\}$ & $B_{3}$ \\
\hline
\end{tabular}

Table 4: Algebras $\mathscr{A}_{J}$ of finite cluster type (non simply-laced case).

Acknowledgements. This paper was written during a stay at the Mathematisches Forschungsinstitut Oberwolfach in July-August 2006. We are very greatful to this institution for its support, its hospitality and for providing ideal working conditions. Some preliminary work was done while B. Leclerc was participating in the program Algebraic Combinatorics at the Mittag-Leffler Institute (Stockholm, April 2005), and in the program Group Representation Theory at the Centre Bernoulli (Lausanne, June 2005). He wants to thank the organizers of these programs for inviting him. 


\section{References}

[1] M. Auslander, S. Smalø, Almost split sequences in subcategories, J. Algebra, 69 (1981), 426-454.

[2] M. Auslander, I. Reiten, Homologically finite subcategories, in "Representations of Algebras and other topics", pp.1-42, Ed. H. Tachikawa, S. Brenner, London Mathematical Society Lecture Notes Series, 168, Cambridge University Press 1992.

[3] R. Bautista, R. Martinez, Representations of partially ordered sets and 1-Gorenstein Artin algebras, in "Proceedings, Conference on Ring Theory, Antwerp, 1978”, pp. 385-433, Dekker, 1979.

[4] A. Berenstein, S. Fomin, A. Zelevinsky, Cluster algebras III. Upper bounds and double Bruhat cells. Duke Math. J., 126 (2005), 1-52.

[5] A. Borel, Linear algebraic groups, 2nd Enlarged Edition, Springer 1991.

[6] N. Bourbaki, Groupes et algèbres de Lie, Chap. 4, 5, 6, Hermann 1968.

[7] A. Buan, O. Iyama, I. Reiten and J. Scott, Cluster structures for 2-Calabi-Yau categories and unipotent groups, arXiv:math.RT/0701557.

[8] A. Buan, R. Marsh, M. Reineke, I. Reiten and G. Todorov, Tilting theory and cluster combinatorics, Adv. Math. 204 (2006), 572-618.

[9] S. Fomin, A. Zelevinsky, Double Bruhat cells and total positivity, J. Amer. Math. Soc. 12 (1999), 335-380.

[10] S. Fomin, A. Zelevinsky, Cluster algebras. I. Foundations, J. Amer. Math. Soc. 15 (2002), 497-529.

[11] S. Fomin, A. Zelevinsky, Cluster algebras II. Finite type classification, Invent. Math., 154 (2003), 63-121.

[12] C. Geiß, B. Leclerc, J. Schröer, Semicanonical bases and preprojective algebras, Ann. Sci. Ecole Norm. Sup., 38 (2005), 193-253.

[13] C. Geiß, B. Leclerc, J. Schröer, Verma modules and preprojective algebras, Nagoya Math. J., 182 (2006), 241-258.

[14] C. Geiß, B. Leclerc, J. Schröer, Auslander algebras and initial seeds for cluster algebras, arXiv:math.RT/0506405, J. London Math. Soc. (to appear).

[15] C. Geiß, B. Leclerc, J. Schröer, Rigid modules over preprojective algebras, Invent. Math., 165 (2006), 589-632.

[16] C. Geiß, B. Leclerc, J. Schröer, Semicanonical bases and preprojective algebras II: A multiplication formula, arXiv:math.RT/0509483, Compositio Math. (to appear).

[17] C. Geiß, B. Leclerc, J. Schröer, Rigid modules over preprojective algebras II: the Kac-Moody case, arXiv:math.RT/0703039

[18] C. Geiß, B. Leclerc, J. Schröer, Rigid modules over preprojective algebras III: quasi-hereditary structures on endomorphism algebras, (in preparation).

[19] C. Geiß, J. Schröer, Extension-orthogonal components of preprojective varieties, Trans. Amer. Math. Soc., 357 (2005), 1953-1962.

[20] M. Gekhtman, M. Shapiro, A. Vainshtein, Cluster algebras and Poisson geometry, Moscow Math. J, 3 (2003), 899-934. 
[21] D. Happel, Triangulated categories in the representation theory of finite-dimensional algebras. London Mathematical Society Lecture Note Series 119. Cambridge University Press, Cambridge (1988), $\mathrm{x}+208 \mathrm{pp}$.

[22] M. Hoshino, On splitting torsion theories induced by tilting modules, Comm. Alg., 11 (1983), 427439.

[23] M. Kashiwara, Y. Saito, Geometric construction of crystal bases, Duke Math. J. 89 (1997), 9-36.

[24] B. Keller, On triangulated orbit categories, Doc. Math. 10 (2005), 551-581 (electronic).

[25] M. Kleiner, Approximations and almost split sequences in homologically finite subcategories, J. Algebra, 198 (1997), 135-163.

[26] V. Lakshmibai, N. Gonciulea, Flag varieties, Travaux en cours 63, Hermann 2001.

[27] G. Lusztig, Canonical bases arising from quantized enveloping algebras, J. Amer. Math. Soc., 3 (1990), 447-498.

[28] G. Lusztig, Quivers, perverse sheaves, and quantized enveloping algebras. J. Amer. Math. Soc., 4 (1991), 365-421.

[29] G. Lusztig, Introduction to quantum groups, Birkhäuser 1993.

[30] A. Postnikov, Total positivity, grassmannians and networks, arXiv:math.CO/0609764

[31] C. M. Ringel, The preprojective algebra of a quiver, Algebras and Modules II, (Geiranger, 1996), CMS Conf. Proc., 24, AMS (1998), 467-480.

[32] J. Scott, Grassmannians and cluster algebras, Proc. London Math. Soc., 92 (2006), 345-380.

[33] A. Seven, Recognizing cluster algebras of finite type, Electron. J. Combin. 14 (2007), no. 1, Research Paper 3, 35 pp. (electronic).

Christof GeISS : $\quad$ Instituto de Matemáticas,

Universidad Nacional Autónoma de México

04510 México D.F., México

email : christof@math.unam.mx

Bernard LECLERC : Université de Caen, LMNO UMR 6139

14032 Caen cedex, France

email : leclerc@math.unicaen.fr

Jan SCHRÖER : $\quad$ Mathematisches Institut, Universität Bonn,

Beringstr. 1, D-53115 Bonn, Germany

email : schroer@math.uni-bonn.de 